\title{
Use of Acupuncture for the Treatment of Sports-Related Injuries in Athletes: A Systematic Review of Case Reports
}

\author{
Ji-Won Lee ${ }^{1}$, Jun-Hwan Lee ${ }^{2,3, *(\mathbb{D})}$ and Song-Yi Kim ${ }^{1, *(\mathbb{D})}$ \\ 1 College of Korean Medicine, Gachon University, Seongnam 13120, Korea; behelia11@gmail.com \\ 2 Clinical Medicine Division, Korea Institute of Oriental Medicine, Daejeon 34054, Korea \\ 3 Korean Medicine Life Science, University of Science \& Technology (UST), Campus of Korea Institute of \\ Oriental Medicine, Daejeon 34054, Korea \\ * Correspondence: omdjun@kiom.re.kr (J.-H.L.); songyi@gachon.ac.kr (S.-Y.K.); Tel.: +82-42-868-9693 (J.-H.L.); \\ +82-31-750-8826 (S.-Y.K.)
}

Received: 28 September 2020; Accepted: 29 October 2020; Published: 6 November 2020

\begin{abstract}
Acupuncture is one of the representative complementary and alternative medicine treatments used for various types of pain. This systematic review summarized and analyzed clinical case reports/series utilizing acupuncture for treating sports injuries in athletes, thereby providing the basis for further research to establish clinical evidence on acupuncture treatment in sports medicine. A comprehensive literature search was conducted in Embase including MEDLINE up to 21 August 2019 without language and publication date restrictions. Due to the heterogeneity of each study, explanatory and descriptive analyses were performed. As a result, in each case report/series, it was confirmed that acupuncture was applied for treating various types of sports injuries experienced by athletes. Acupuncture can help relieve short-term pain and recover from dysfunction and has been used as a useful, noninvasive, and conservative modality for managing sports injuries such as lateral meniscus rupture, femoral acetabular impingement, ganglion cysts, and sports hernia. In addition, acupuncture has been suggested as a treatment worth trying for diseases such as yips and delayed onset muscle soreness. The included cases showed some potential of acupuncture in the treatment of various types of sports injuries, beyond pain control in musculoskeletal disorders. However, considering that this review was based on case reports/series, a limited understanding of the clinical value of acupuncture in athletes is required. In the future, more specific research questions and hypotheses should be addressed to generate evidence based on experimental research.
\end{abstract}

Keywords: sports injury; athlete; acupuncture; case report; case series; review

\section{Introduction}

In recent years, exercise has served a multitude of functions, in addition to promoting health [1]. Regular exercise not only improves an individual's health, but is also considered as a "therapy" for people from all walks of life, such as the pediatric and elderly population, those with chronic diseases, or those with obesity [2,3]. Exercise generally has obvious benefits, but it also requires proper techniques, strength, and frequency [1]. At present, as the population of sports enthusiasts increases, sports injuries have become a prevalent affliction [4]. Musculoskeletal disorders account for most sports injuries and tend to recur frequently $[5,6]$. Although rare compared to musculoskeletal diseases, sports medicine encompasses various kinds of diseases that preclude sports activities, including psychological issues [7].

In comparison to recreational athletes or non-athletes, elite athletes are deemed to possess more specific goals and are more motivated for improving performance [8]. It is natural that sports injuries 
are very crucial to elite athletes because they directly affect their careers and economic profit. Therefore, an athlete-centered, long-term, return-to-play (RTP) strategy for those with sports injuries is deemed to be of utmost priority [9]. The objective of an athlete's treatment and rehabilitation is to return to their respective sports with minimal performance loss, whereas that of non-athletes is to return to normal daily activities [9]. Accordingly, athletes' sports injuries often lead to complicated considerations in the doctors' decision-making process [10].

Regarding therapeutic approach for sports injuries, it is important to consider the patient background, including the type of sport and nature of the injury (acute or overuse), or their usual training or match schedules, as well as whether they belong to professional or recreational sports [11]. Thus, practitioners need to know the wealth of clinical evidence about sports-specific profiles and various treatment options [12]. Experimental studies, including randomized controlled trials (RCTs) or controlled clinical trials (CCTs), commonly provide valuable evidence [13] but may have limitations, especially in athletes. Although little has been studied about the motivations of elite athletes to participate in clinical studies, the benefits, generally considered being patients' motivations in clinical study, including access to health care and personal benefit, altruism, and monetary incentives [14], may not be attractive to elite athletes. This could be the reason why experiments conducted on athletes were mainly uncontrolled trials involving the participation of an entire team or experiments conducted with young nonprofessional athletes or in a heterogeneous group of athletes. Another limitation is that the research inclusion criteria required by RCTs are often too restrictive for a wide range of sports injuries, and the statistical results of RCTs can often be regarded as ignoring or equalizing the participants' background characteristics [13]. A case report is a detailed report of an individual patient's diagnosis, treatment, response to treatment, and follow-up, while a case series aggregates individual cases who received similar treatments into one report [15]. Essentially, clinical case reports or series (case reports/series) not only belong at the bottom of the hierarchy of evidence-based medicine (EBM), but also provide a lower causal relationship compared to RCTs [16]. However, they can provide important and beneficial clues about potential treatments for athletes. In addition, case reports/series are the primary sources of information on new treatment options, responses to treatment, details of management, long-term follow-up, and unexpected adverse events (AEs) of treatment [17].

Acupuncture is a nonpharmaceutical therapy known to induce pain control, especially in the musculoskeletal system [18]. Various therapeutic effects of acupuncture are associated with central and systemic mechanisms involving the brain or autonomic nervous system as well as local effects at the acupuncture site [19]. Previous studies have shown that acupuncture changes the levels of neurotransmitters or hormones such as beta-endorphin, dopamine, serotonin, and cortisol [20-22] and has a specific effect on the limbic system or emotional area of the brain $[19,23]$. Also, other studies have further elucidated that acupuncture may be beneficial in treating chronic pain with depression [24], drug addiction [25], and degenerative disorders such as Alzheimer disease [26].

At present, with regard to potential advantages of acupuncture, case reports/series on acupuncture in athletes have been published steadily [27-30]. However, limited reviews with a comprehensive perspective on the use of acupuncture in athletes have been reported. Therefore, this systematic review aimed to summarize and analyze clinical case reports/series utilizing acupuncture for treating sports injuries of athletes, thereby providing the basis for further research to establish clinical evidence and expand the scope of acupuncture treatment in sports medicine. Furthermore, this study sought to provide useful insights for practitioners willing to utilize acupuncture in the clinical practice.

\section{Materials and Methods}

\subsection{Search Strategy}

To establish the search strategy that could comprehensively include clinical studies of athletes receiving acupuncture treatment for sports injuries, taking into account the search term sensitivity and specificity, a preliminary search was conducted in PubMed (https://pubmed.ncbi.nlm.nih.gov/) 
and domestic databases (Research Information Sharing Service (RISS, http://www.riss.kr/), National Digital Science Library (NDSL, http://www.ndsl.kr/), and Oriental Medicine Advanced Searching Integrate System (OASIS, https://oasis.kiom.re.kr/)). Based on these results, the following search terms were selected. The search terms used consisted of a combination of traditional Korean medicine treatment ("traditional Korean medicine", "traditional Chinese medicine", "acupuncture", "herb*", "moxibustion", "cupping", or "pharmacopuncture" ("aqua acupuncture", "herb* acupuncture", or "acupoint injection")) and sports injury ((("wound", "medicine", or "injury") and ("athletic" or "sport")), "athletic injury", "sports injury", or "sports medicine"). Finally, a literature search was conducted in Embase (www.embase.com) including MEDLINE up to 21 August 2019 without language and publication date restrictions. Furthermore, the reference lists of related reviews were screened to ensure that there were no missing studies. We also manually identified additional records through other sources, including Google Scholar. All searched studies were listed and organized using Microsoft Excel (Microsoft, Redmond, WA, USA).

\subsection{Study Selection, Data Extraction, and Data Analysis}

The inclusion criteria were clinical case reports or series that studied the effects of treatment, including acupuncture, on sports injuries of athletes. To clarify this inclusion criterion, we reviewed and established several definitions among the authors. Firstly, we included manual acupuncture (MA), electroacupuncture (EA), auricular acupuncture, laser acupuncture (LA), acupressure, catgut embedding, dry needling, and transcutaneous electrical nerve stimulation (TENS) in the category of "acupuncture". It is controversial whether TENS can be classified as a type of acupuncture; hence, we only included it in the analysis when the TENS was applied to the acupuncture points. Secondly, we defined case reports/series as a descriptive study based on uncontrolled observations containing detailed information on the diagnosis, treatment, response to treatment, and follow-up of individual patients. Several steps were taken based on the following exclusion criteria to select a study whose study design was a case report or a case series, regardless of the number of cases in an individual patient. The exclusion criteria were as follows: (1) Studies not targeted for humans (i.e., in vitro studies or animal studies), (2) research that did not collect original primary data (review, protocol, editorial, letter, etc.), (3) cross-sectional study or prevalence research, (4) experimental study with control (i.e., randomized controlled trials, controlled clinical trials), (5) no acupuncture-related treatment interventions (defined in advance) were used, (6) not targeted for athletes, (7) full text of the research results were not provided (i.e., conference poster abstract), and (8) studies not written in English, Chinese, or Korean. Since case reports/series are mostly retrospective observational studies, in many cases, different combination treatments are commonly used together, and it is ambiguous to distinguish which treatment was employed as the primary or adjuvant treatment. Therefore, we included all studies where the use of acupuncture as the main treatment was uncertain, but conducted a rigorous evaluation in the screening process to ensure that acupuncture was used for treatment in each individual study.

The records were screened accordingly and independently by two authors (J.-W.L. and S.-Y.K.) based on the title, abstract, or full text. First, all duplicate records belonging to the same title and author were removed. Grounded on the above inclusion/exclusion criteria, studies that were unrelated to sports medicine or studies that focused on doping, which fall into the category of sports medicine but failed to satisfy the inclusion criteria for this study, were first excluded for convenience. Next, the studies that corresponded to the exclusion criteria were removed to finally screen appropriate studies for our review analysis.

\subsection{Data Extraction and Data Analysis}

Data extraction was performed by one author (J.-W.L.) using predefined data extraction forms and then reviewed by another author (S.-Y.K.). If there was a discrepancy or further discussion was needed, it was resolved by another investigator (J.-H.L.), who was not involved in the extraction and review process. The data were extracted by classifying the following categories. These categories were 
modified based on some of the topic and checklist items in the CAse REport (CARE) guideline [31]: patient information, therapeutic intervention, follow-up, and outcomes were extracted. In the "patient information," main concerns and symptoms of the patient including duration of the disorder, relevant past interventions, and patients' demographic data such as type of sports, age, and gender were collected. For "therapeutic intervention", types of acupuncture and acupuncture points, types of co-intervention, administration of intervention (such as treatment duration and number of treatment sessions), and changes in intervention with rationale were extracted. In relation to "follow-up and outcomes", the outcome measures used in each study, their results, and AEs were collected, and information on RTP, recurrence, and conclusions from the authors of each study were summarized and analyzed.

The included sports were analyzed based on static components during competition according to the sports classification by Mitchell et al. [32]. If such sports did not quite match up to Mitchell et al.'s classification, we classified the sports in a consensus discussion between the authors.

Our study was not homogeneous enough to quantitatively synthesize the results of each individual study as not limited to a specific disease and did not include a control group. Therefore, it was not possible to evaluate the effectiveness by estimating the size of the effect or by statistically pooling and calculating the results between studies, and only descriptive and narrative results of qualitative analysis on the role of "acupuncture" described in each individual study were provided.

\section{Results}

Our screening process is illustrated in Figure 1. Initially, 1954 potentially relevant articles were searched through the database, and 36 additional records were identified by manual searching. After removing duplicates $(n=31)$ from a total of 1990 records identified using the search strategy, 1959 studies were screened in view of the title, abstract, or full text, and 1937 were discarded (1558 studies were unrelated to sports medicine; 142 studies were not primary studies; 100 studies were experimental studies; 30 studies were not related to intervention using acupuncture points, etc.).

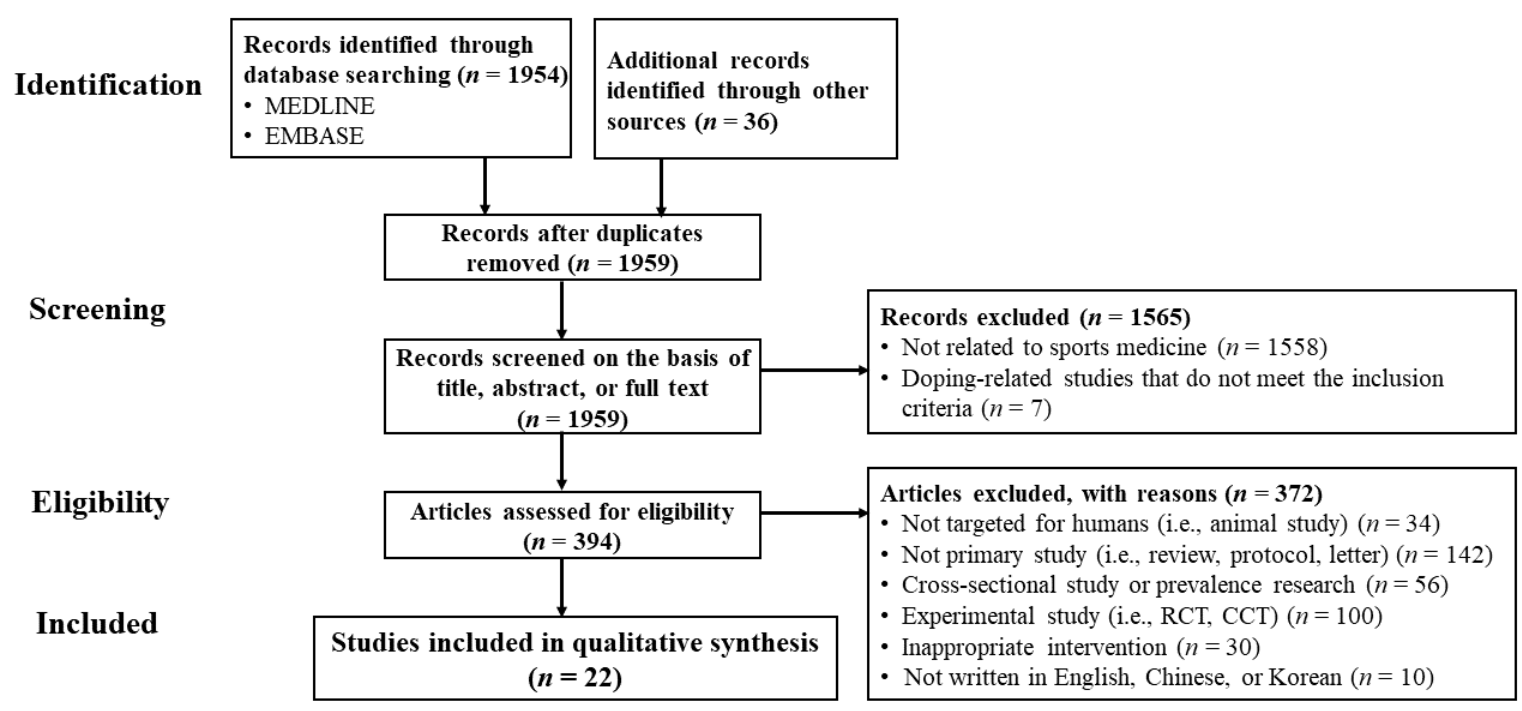

Figure 1. Flow diagram. CCT, controlled clinical trial; RCT, randomized controlled trial.

Finally, 22 case reports/series were included in our review [27-30,33-50]. The summary of the characteristics of the inclusion studies based on the items in the CARE checklist is shown in Table 1. 
Table 1. Summary of included case reports/series.

\begin{tabular}{|c|c|c|c|c|c|c|c|c|c|c|c|c|c|c|}
\hline \multicolumn{3}{|c|}{ Study } & \multicolumn{3}{|c|}{ Demographic Information } & \multicolumn{2}{|c|}{$\begin{array}{l}\text { Main Concerns and Symptoms } \\
\text { of the Patient }\end{array}$} & \multirow{2}{*}{$\begin{array}{c}\text { Medical History } \\
\text { Previous } \\
\text { Interventions }\end{array}$} & \multirow{2}{*}{$\begin{array}{c}\begin{array}{c}\text { Administration } \\
\text { of Intervention }\end{array} \\
\begin{array}{c}\text { (1) Interventions } \\
\text { (2) Duration } \\
\text { (3) No. of sessions }\end{array}\end{array}$} & \multicolumn{5}{|c|}{ Follow-up and Outcomes } \\
\hline No & $\begin{array}{l}\text { Study } \\
\text { ID }\end{array}$ & $\begin{array}{l}\text { Coun. } \\
\text { (Lang) }\end{array}$ & $\begin{array}{l}\text { Sports } \\
\text { type }\end{array}$ & Age (yr) & $\begin{array}{l}\text { No. of } \\
\text { Cases }\end{array}$ & Disorders & Duration & & & $\begin{array}{l}\text { Outcome } \\
\text { measures }\end{array}$ & Results & $\begin{array}{l}\text { Adverse } \\
\text { events }\end{array}$ & $\begin{array}{l}\text { (1) Return to play } \\
\text { (2) Recurrence } \\
\text { (f/u) }\end{array}$ & $\begin{array}{l}\text { Authors' } \\
\text { comments for } \\
\text { acupuncture } \\
\text { treatments }\end{array}$ \\
\hline \multicolumn{15}{|c|}{ Diseases of the musculoskeletal system and connective tissue } \\
\hline 1 & $\begin{array}{l}\text { Dlin, } \\
1980 \\
{[33]}\end{array}$ & $\begin{array}{l}\text { Israel } \\
\text { (Eng.) }\end{array}$ & ND & $\begin{array}{c}12-44 \\
(\text { mean }= \\
2(5)\end{array}$ & 22 & $\begin{array}{l}\text { Musculotendinous } \\
\text { syndromes of the } \\
\text { chronic exertion } \\
\text { type (shoulder, } \\
\text { knee, anterior } \\
\text { tibia, back pain) }\end{array}$ & $\begin{array}{c}\text { Few } \\
\text { wks-several } \\
\text { mon }\end{array}$ & $\begin{array}{l}\text { At least a } 2 \text { to } 3 \\
\text { wk period: [all } \\
\text { patients] heat } \\
\text { application, rest; } \\
\text { [as needed] oral } \\
\text { anti-inflammatory } \\
\text { medication } \\
\text { (indomethacin), } \\
\text { physiotherapy } \\
\text { (ultrasound, or } \\
\text { local steroid } \\
\text { injections) }\end{array}$ & $\begin{array}{l}\text { (1) TENS } \\
\text { (2) ND } \\
\text { (3) } 2-6\end{array}$ & $\begin{array}{l}\text { (1) Pain } \\
\text { questionnaire } \\
\text { (2) Pain } \\
\text { grade }\end{array}$ & $\begin{array}{c}\text { (1) Complete pain } \\
\text { relief }(n=1(5), \\
\text { good (>50\%) } \\
\text { reduction) } \\
(n=(4) \text {, fair } \\
(30-50 \% \\
\text { reduction) } \\
(n=(1), \text { poor } \\
(<30 \% \text { reduction) } \\
(n=(2) \\
(2) \text { Grade } 0 \\
(n=17), 2 \text { grades } \\
\text { reduction (good } \\
\text { result) }(n=(2), \\
1 \text { grade reduction } \\
\text { (fair result) } \\
(n=(1), \text { No } \\
\text { changes }(n=(2)\end{array}$ & None & $\begin{array}{l}\text { (1) } 18 \text { of } 22 \\
\text { subjects returned } \\
\text { to full sports } \\
\text { activity } \\
\text { (2) 10-day-2-mon } \\
\text { after return to full } \\
\text { sports activity } \\
(n=6)\end{array}$ & $\begin{array}{l}\text { TENS stimulation } \\
\text { of acupuncture } \\
\text { points may be } \\
\text { effective in the } \\
\text { therapy of painful } \\
\text { sports injuriries of } \\
\text { the athletic } \\
\text { exertion type. }\end{array}$ \\
\hline 2 & $\begin{array}{l}\text { Sternfeld, } \\
1992 \\
{[34]}\end{array}$ & $\begin{array}{l}\text { Israel } \\
\text { (Eng.) }\end{array}$ & $\begin{array}{l}\text { Long-distanc } \\
\text { running }\end{array}$ & nce 16 & 1 & $\begin{array}{l}\text { Runner's stitch } \\
\text { syndrome }\end{array}$ & Several mon & $\begin{array}{l}\text { Analgesics and } \\
\text { NSAIDs }\end{array}$ & $\begin{array}{c}\text { (1) MA } \\
\text { (2) } 31 \text {-day [1/day } \\
\text { (3-day)+1/wk } \\
\text { (4-wk)] } \\
\text { (3) } 7\end{array}$ & Symptoms & $\begin{array}{l}\text { Complete and } \\
\text { permanent } \\
\text { recovery }\end{array}$ & ND & $\begin{array}{l}\text { (1) Resuming all } \\
\text { activities and } \\
\text { achievements in } \\
\text { competitive } \\
\text { sports } \\
\text { (2) None } \\
\text { (several wks) }\end{array}$ & $\begin{array}{l}\text { We suggest } \\
\text { acupuncture as a } \\
\text { preferred therapy } \\
\text { for cases involving } \\
\text { runner's stitch } \\
\text { syndrome. }\end{array}$ \\
\hline 3 & $\begin{array}{l}\text { Seplow, } \\
1996 \\
{[35]}\end{array}$ & $\begin{array}{l}\text { USA } \\
\text { (Eng.) }\end{array}$ & Basketball & 17 & 1 & $\begin{array}{l}\text { Lateral meniscus } \\
\text { injury (a tear of } \\
\text { the posterior } \\
\text { horn of the lateral } \\
\text { meniscus) }\end{array}$ & 3-day & $\begin{array}{l}\text { Ice, rest, NSAIDs } \\
\text { (2-day) }\end{array}$ & $\begin{array}{l}\text { (1) EA + others } \\
\text { (2) Initial 2-wk } \\
\text { (3) ND }\end{array}$ & Symptoms & $\begin{array}{l}\text { Walk without } \\
\text { limp or pain and } \\
\text { range of knee } \\
\text { flexion returned } \\
\text { to } 130^{\circ}\end{array}$ & ND & $\begin{array}{l}\text { (1) Return to } \\
\text { training in 5-wks, } \\
\text { full participation } \\
\text { in 8-wks } \\
\text { (2) ND }\end{array}$ & $\begin{array}{l}\text { When tears are } \\
\text { diagnosed, } \\
\text { conservative } \\
\text { methods may be } \\
\text { effective in treating } \\
\text { these ethlletes and } \\
\text { may in fact be more } \\
\text { effective in } \\
\text { many cases. }\end{array}$ \\
\hline
\end{tabular}


Table 1. Cont.

\begin{tabular}{|c|c|c|c|c|c|c|c|c|c|c|c|c|c|c|}
\hline \multicolumn{3}{|c|}{ Study } & \multicolumn{3}{|c|}{ Demographic Information } & \multicolumn{2}{|c|}{$\begin{array}{c}\text { Main Concerns and Symptoms } \\
\text { of the Patient }\end{array}$} & \multirow[b]{2}{*}{$\begin{array}{l}\text { Medical History } \\
\\
\\
\text { Pelvic/lower } \\
\text { extremity } \\
\text { chiropractic } \\
\text { manipulation, } \\
\text { interferential } \\
\text { current, } \\
\text { cryotherapy } \\
\text { (3-day) }\end{array}$} & \multirow[b]{2}{*}{$\begin{array}{l}\begin{array}{c}\text { Administration } \\
\text { of Intervention }\end{array} \\
\\
\\
\text { (1) MA + others } \\
\text { (2) 5-day } \\
\text { (3) } 5\end{array}$} & \multicolumn{5}{|c|}{ Follow-up and Outcomes } \\
\hline 4 & $\begin{array}{l}\text { Hoven, } \\
2000 \\
{[36]}\end{array}$ & $\begin{array}{l}\text { USA } \\
\text { (Eng.) }\end{array}$ & Ski & 19 & 1 & $\begin{array}{l}\text { Peroneal nerve } \\
\text { entrapment and } \\
\text { ankle sprain }\end{array}$ & ND & & & Symptoms & $\begin{array}{l}\text { Pain and } \\
\text { weakness } \\
\text { alleviated after } \\
\text { 3-day of care }\end{array}$ & ND & (1), (2) ND & $\begin{array}{l}\text { It appears that } \\
\text { chiropractic } \\
\text { manipulation of the } \\
\text { lumbar, pelvis and } \\
\text { lower extremity, } \\
\text { combined with } \\
\text { interferential } \\
\text { current, } \\
\text { cryotherapy, } \\
\text { acupuncture and } \\
\text { trigger point } \\
\text { therapy played a } \\
\text { significant role in } \\
\text { alleviating the } \\
\text { peroneal } \\
\text { neuropathy } \\
\text { experienced by this } \\
\text { elite } \\
\text { nationior } \\
\text { national skier. }\end{array}$ \\
\hline 5 & $\begin{array}{c}\text { Young, } \\
2005 \\
{[38]}\end{array}$ & $\begin{array}{l}\text { UK } \\
\text { (Eng.) }\end{array}$ & Rugby & $\begin{array}{l}\# 1: 19 ; \\
\# 2: 32\end{array}$ & 2 & $\begin{array}{l}\text { \#1: Calf } \\
\text { strain/mild } \\
\text { chronic } \\
\text { compartment } \\
\text { syndrome; \#2: } \\
\text { Tennis } \\
\text { elbow/muscle } \\
\text { tightness }\end{array}$ & $\begin{array}{l}\text { \#1: 6-mon; } \\
\text { \#2: ND }\end{array}$ & ND & $\begin{array}{l}\text { (1) } \mathrm{EA}+\mathrm{MA}+ \\
\text { others } \\
\text { (2) \#1: 6-wk } \\
\text { \#2: 4-wk (2/wk) } \\
\text { (3) \#1: } 12 ; \# 2: 8\end{array}$ & Symptoms & $\begin{array}{l}\# 1 \text { : Completion of } \\
\text { the full training } \\
\text { without pain \#2: } \\
\text { Training at ease }\end{array}$ & ND & $\begin{array}{l}\text { (1)\#1: Playing } \\
\text { games without } \\
\text { pain; \#2: Playing } \\
\text { at ease } \\
\text { (2) ND }\end{array}$ & $\begin{array}{l}\text { Even though using } \\
\text { acupuncture is } \\
\text { very effective in } \\
\text { treatment, there } \\
\text { comes a time in the } \\
\text { treatment of an } \\
\text { injury when you } \\
\text { will need to open } \\
\text { the channels } \\
\text { physically to allow } \\
\text { qi and blood to flow } \\
\text { smoothly and to } \\
\text { return the } \\
\text { shortened muscle } \\
\text { back to its } \\
\text { normal length. }\end{array}$ \\
\hline 6 & $\begin{array}{l}\text { Martínez- } \\
\text { Silvestrini, } \\
2006 \\
{[39]}\end{array}$ & $\begin{array}{l}\text { Puerto } \\
\text { Rico } \\
\text { (Eng.) }\end{array}$ & ND & 11 & $1^{*}$ & $\begin{array}{l}\text { CRPS after lower } \\
\text { extremity trauma } \\
\text { and clinical } \\
\text { depression }\end{array}$ & Few wks & $\begin{array}{l}\text { (For CRPS } \\
\text { treatment) } \\
\text { Hydrotherapy, } \\
\text { electrical } \\
\text { stimulation, } \\
\text { ultrasound to the } \\
\text { Achilles tendon, } \\
\text { stretching, active } \\
\text { assisted range of } \\
\text { motion, weight } \\
\text { bearing, opioid } \\
\text { analgesics, } \\
\text { corticosteroids }\end{array}$ & $\begin{array}{l}\text { (1) MA + others } \\
\text { (2) 3-mon } \\
\text { (3) ND }\end{array}$ & Symptoms & $\begin{array}{l}\text { The clinics } \\
\text { discharge } \\
\text { without any } \\
\text { deficits }\end{array}$ & ND & (1), (2) ND & $N D$ \\
\hline
\end{tabular}


Table 1. Cont

\begin{tabular}{|c|c|c|c|c|c|c|c|c|c|c|c|c|c|c|}
\hline \multicolumn{3}{|c|}{ Study } & \multicolumn{3}{|c|}{ Demographic Information } & \multicolumn{2}{|c|}{$\begin{array}{c}\text { Main Concerns and Symptoms } \\
\text { of the Patient }\end{array}$} & \multirow{2}{*}{$\begin{array}{c}\text { Medical History } \\
\text { ND }\end{array}$} & \multirow{2}{*}{$\begin{array}{c}\begin{array}{c}\text { Administration } \\
\text { of Intervention }\end{array} \\
\\
\text { (1) EA + others } \\
\text { (2) ND (one } \\
\text { course comprised } \\
7 \text {-day) } \\
\text { (3) ND }\end{array}$} & \multicolumn{5}{|c|}{ Follow-up and Outcomes } \\
\hline 7 & $\begin{array}{l}\text { Yan, } \\
2008 \\
{[41]}\end{array}$ & $\begin{array}{l}\text { China } \\
\text { (Chin.) }\end{array}$ & Football & $\underset{21}{\operatorname{Mean}=}$ & $19^{* *}$ & $\begin{array}{l}\text { Sports injury in } \\
\text { knee joint medial } \\
\text { collateral } \\
\text { ligament }\end{array}$ & ND & & & $\begin{array}{l}\text { (1) Recovery } \\
\text { time } \\
\text { (2) } \\
\text { Therapeutic } \\
\text { effect } \\
\text { (3) The } \\
\text { overall } \\
\text { efficiency } \\
\end{array}$ & $\begin{array}{l}\text { (1) Rapid (23\%); } \\
\text { moderate (50\%) } \\
\text { (2) Recovered } \\
\text { (40\%); partly } \\
\text { recovered (53\%) } \\
\text { (3) } 93 \%\end{array}$ & $\mathrm{ND}$ & (1), (2) ND & $N D$ \\
\hline 8 & $\begin{array}{l}\text { Osborne, } \\
2010 \\
{[42]}\end{array}$ & $\begin{array}{l}\text { UK } \\
\text { (Eng.) }\end{array}$ & Volleyball & $\begin{array}{l}23-27 \\
\underset{(\text { mean }}{2(5)}\end{array}$ & 4 & $\begin{array}{c}\text { Anterior/ } \\
\text { anterolateral } \\
\text { shoulder pain }{ }^{(1)}\end{array}$ & $\begin{array}{l}\text { \#1: } 2 \text { mon.; } \\
\text { \#2: } 6 \text { yr.; \#3: } \\
\text { 18 mon.; \#4: } \\
\text { 6-mon }\end{array}$ & $\begin{array}{l}\text { Conservative } \\
\text { treatment (\#1: } \\
\text { Thera band, } \\
\text { stretching; \#2, \#3: } \\
\text { ND; \#4: exercises, } \\
\text { stretching) }\end{array}$ & $\begin{array}{l}\text { (1) MA + others } \\
\text { (2) 1-mon-long } \\
\text { intense } \\
\text { competitive } \\
\text { phase } \\
\text { (3) \#1, \#3, \#4: 1; \#2: } \\
2\end{array}$ & $\begin{array}{c}(1) \\
\text { Functional } \\
\text { assessment } \\
\text { (MPQ) } \\
\text { (2) ROM } \\
\text { (3) Verbal } \\
\text { pain scores } \\
\text { (4) Muscle } \\
\text { strength }\end{array}$ & $\begin{array}{l}\text { (1)-(4) All scores } \\
\text { improved }\end{array}$ & ND & $\begin{array}{l}\text { (1) Continuing } \\
\text { overhead } \\
\text { activities } \\
\text { (2) ND }\end{array}$ & $\begin{array}{l}\text { These cases support } \\
\text { the use of drry } \\
\text { needling in elite } \\
\text { athletes during a } \\
\text { competitive phase } \\
\text { with short-term } \\
\text { pain relief and } \\
\text { improved function } \\
\text { in shoulder } \\
\text { injuries. }\end{array}$ \\
\hline 9 & $\begin{array}{l}\text { Pearcey, } \\
2013 \\
{[46]}\end{array}$ & $\begin{array}{l}\text { Canada } \\
\text { (Eng.) }\end{array}$ & ND & 31 & 1 & $\begin{array}{l}\text { Exertional } \\
\text { rhabdomyolysis }\end{array}$ & Few days & $\begin{array}{l}\text { Intravenous } \\
\text { hydration with } \\
\text { sodium } \\
\text { bicarbonate and } \\
\text { blood work } \\
\text { [3 days] }\end{array}$ & $\begin{array}{l}\text { (1) MA + others } \\
\text { (2) } 2 \text { day } \\
\text { (3) ND }\end{array}$ & CK levels & Declined by $45 \%$ & $\mathrm{ND}$ & $\begin{array}{l}\text { (1) } 1 \text { mon. after } \\
\text { the diagnosis, the } \\
\text { subject slowly } \\
\text { returned to } \\
\text { high-intensity } \\
\text { resistance } \\
\text { training }(>2 / \text { wk.) } \\
\text { without any } \\
\text { complications } \\
\text { (2) ND }\end{array}$ & $N D$ \\
\hline 10 & $\begin{array}{l}\text { Woitzik, } \\
2013 \\
{[47]}\end{array}$ & $\begin{array}{l}\text { Canada } \\
\text { (Eng.) }\end{array}$ & Cycle & 45 & 1 & $\begin{array}{l}\text { Ganglion cyst of } \\
\text { the foot }\end{array}$ & 10-day & ND & $\begin{array}{l}\text { (1) EA } \\
\text { (2) } 4 \text {-wk }(1 / \mathrm{wk}) \\
\text { (3) } 4\end{array}$ & Symptoms & $\begin{array}{l}\text { Resolution of the } \\
\text { cyst (11 day after } \\
\text { final treatment) }\end{array}$ & $\mathrm{ND}$ & (1), (2) ND & $\begin{array}{l}\text { EA may be a novel } \\
\text { and non-invasive } \\
\text { conservative } \\
\text { approach for the } \\
\text { treatment of } \\
\text { ganglion cysts. }\end{array}$ \\
\hline 11 & $\begin{array}{l}\text { Morimoto, } \\
2013 \\
{[45]}\end{array}$ & $\begin{array}{l}\text { Japan } \\
\text { (Eng.) }\end{array}$ & $\begin{array}{l}\text { Various } \\
\text { sports }\end{array}$ & $\begin{array}{c}17-77 \\
(\text { mean } \\
39)\end{array}$ & 41 & $\begin{array}{l}\text { Jumper's knee, } \\
\text { lateral/medial } \\
\text { epicondylitis, } \\
\text { Achilles } \\
\text { tendinitis, } \\
\text { adductor muscle } \\
\text { injury, rotator cuff } \\
\text { injuries, etc. }{ }^{(3)}\end{array}$ & ND & ND & $\begin{array}{c}\text { (1) LA }+ \text { others } \\
\text { (2) ND } \\
\text { (3) 2-10 (mean = } \\
4 .(1)\end{array}$ & $\begin{array}{l}\text { Pain Relief } \\
\text { Score }\end{array}$ & $\begin{array}{c}5 \text { or less }(n= \\
27,66 \%)\end{array}$ & $\mathrm{ND}$ & (1), (2) ND & $\begin{array}{l}\text { Low-level LA is an } \\
\text { effective treatment } \\
\text { for sports injuries, } \\
\text { particularly } \\
\text { jumper's knee, } \\
\text { tennis elbow and } \\
\text { Achilles tendinitis. }\end{array}$ \\
\hline
\end{tabular}


Table 1. Cont.

\begin{tabular}{|c|c|c|c|c|c|c|c|c|c|c|c|c|c|c|}
\hline \multicolumn{3}{|c|}{ Study } & \multicolumn{3}{|c|}{ Demographic Information } & \multicolumn{2}{|c|}{$\begin{array}{c}\text { Main Concerns and Symptoms } \\
\text { of the Patient }\end{array}$} & \multirow{2}{*}{$\begin{array}{c}\text { Medical History } \\
\\
\text { Self-massage, } \\
\text { OTC NSAIDs }\end{array}$} & \multirow{2}{*}{$\begin{array}{c}\begin{array}{c}\text { Administration } \\
\text { of Intervention }\end{array} \\
\\
\begin{array}{c}\text { (1) } \mathrm{MA}+\text { others } \\
\text { (2) } \mathrm{ND}(1 / \mathrm{wk}) \\
\text { (3) } 2\end{array}\end{array}$} & \multicolumn{5}{|c|}{ Follow-up and Outcomes } \\
\hline 12 & $\begin{array}{l}\text { Gliedt, } \\
2014 \\
{[49]}\end{array}$ & $\begin{array}{l}\text { USA } \\
\text { (Eng.) }\end{array}$ & Basketball & 41 & 1 & $\begin{array}{l}\text { Sub-acute left } \\
\text { elbow } \\
\text { tendinopathy }\end{array}$ & $5 \mathrm{wk}$ & & & Symptoms & $\begin{array}{l}\text { Reduced left } \\
\text { elbow swelling } \\
\text { and left wrist } \\
\text { extensor muscle } \\
\text { hypertonicity, } \\
\text { and no pain }\end{array}$ & ND & $\begin{array}{l}\text { (1) A return to } \\
\text { normal } \\
\text { recreational } \\
\text { athletic activities } \\
\text { including } \\
\text { basketball } \\
\text { (2) None (6-wk) }\end{array}$ & $\begin{array}{l}\text { The patient's } \\
\text { outcomes inticated } \\
\text { a quick resolution } \\
\text { of subjective } \\
\text { complaints and } \\
\text { objective findings } \\
\text { with the chosen } \\
\text { treatment. }\end{array}$ \\
\hline 13 & $\begin{array}{c}\text { Jayaseelan, } \\
2014 \\
{[50]}\end{array}$ & $\begin{array}{c}\text { USA } \\
\text { (Eng.) }\end{array}$ & Running & $\begin{array}{l}\# 1: 70 ; \\
\# 2: 69\end{array}$ & 2 & $\begin{array}{c}\text { Proximal } \\
\text { hamstring } \\
\text { tendinopathy }\end{array}$ & $\begin{array}{l}\text { \#1: } 7 \mathrm{mon} ; \\
\# 2: 5 \mathrm{mon}\end{array}$ & $\begin{array}{l}\text { Eccentric training } \\
\text { of hamstrings, } \\
\text { lumbopelvic } \\
\text { stabilization } \\
\text { exercises, patient } \\
\text { education (2-wk: } \\
\text { "Phase 1" } \\
\text { treatment) }\end{array}$ & 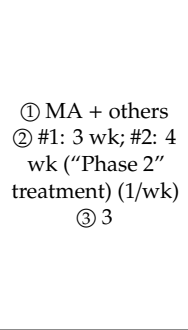 & $\begin{array}{l}\text { (1) Pain } \\
\text { (NRS) } \\
\text { (2) Global } \\
\text { rating of } \\
\text { change } \\
\text { (3) Lower } \\
\text { Extremity } \\
\text { Functional } \\
\text { Scale } \\
\text { (4) Tenderness } \\
\text { (5) Strength }\end{array}$ & $\begin{array}{l}\text { (1)-(5) All score } \\
\text { improvement, } \\
\text { significant } \\
\text { function } \\
\text { improvement }\end{array}$ & ND & $\begin{array}{l}\text { (1) Each patient } \\
\text { returned to } \\
\text { running and } \\
\text { sitting without } \\
\text { symptoms } \\
\text { (2) None }(6 \mathrm{mon})\end{array}$ & $\begin{array}{l}\text { In these } 2 \text { runners, } \\
\text { eccentric loading of of } \\
\text { the hamstrings, } \\
\text { lumbopelvic } \\
\text { stabilization } \\
\text { exercises, and } \\
\text { trigger point dry } \\
\text { needling provided } \\
\text { short- and } \\
\text { long-term pain } \\
\text { reduction and } \\
\text { functional benefits. }\end{array}$ \\
\hline 14 & $\begin{array}{l}\text { MacIntyre, } \\
2015 \\
{[27]}\end{array}$ & $\begin{array}{l}\text { Canada } \\
\text { (Eng.) }\end{array}$ & $\begin{array}{c}\text { Ice } \\
\text { hockey }\end{array}$ & 22 & 1 & FAI & $4 \mathrm{yr}$ & None & $\begin{array}{c}\text { (1) } \mathrm{EA}+\text { others } \\
\text { (2) } 6-\mathrm{wk}(1-2 / \mathrm{wk}) \\
\text { (3) } 8\end{array}$ & Symptoms & $\begin{array}{l}\text { No pain [at rest, } \\
\text { daily activities } \\
\text { (including } \\
\text { exercise), all } \\
\text { stress tests used } \\
\text { for initial } \\
\text { physical } \\
\text { examination]; } \\
\text { despite having } \\
\text { less pain, the hip } \\
\text { scour and FADIR } \\
\text { tests still } \\
\text { provided a hard } \\
\text { end-feel and } \\
\text { palpable click at } \\
\text { extreme ROM }\end{array}$ & ND & $\begin{array}{l}\text { (1) At } 8 \mathrm{wk} \\
\text { (2) None }(6 \mathrm{mon})\end{array}$ & $\begin{array}{l}\text { Conservative } \\
\text { management } \\
\text { utilizizing a } \\
\text { multimodal } \\
\text { approach, as } \\
\text { described in the } \\
\text { case, } \\
\text { lineolld be frearst } \\
\text { line treatment. }\end{array}$ \\
\hline
\end{tabular}


Table 1. Cont.

\begin{tabular}{|c|c|c|c|c|c|c|c|c|c|c|c|c|c|c|}
\hline \multicolumn{3}{|c|}{ Study } & \multicolumn{3}{|c|}{ Demographic Information } & \multicolumn{2}{|c|}{$\begin{array}{c}\text { Main Concerns and Symptoms } \\
\text { of the Patient }\end{array}$} & \multirow{2}{*}{$\begin{array}{c}\text { Medical History } \\
\text { N/A }\end{array}$} & \multirow{2}{*}{$\begin{array}{c}\begin{array}{c}\text { Administration } \\
\text { of Intervention }\end{array} \\
\\
\text { (1) Catgut } \\
\text { embedding } \\
\text { (2) } 90-120 \text { day } \\
\text { (3) } 6\end{array}$} & \multicolumn{5}{|c|}{ Follow-up and Outcomes } \\
\hline 15 & $\begin{array}{l}\text { Chen, } \\
2008 \\
{[40]}\end{array}$ & $\begin{array}{l}\text { China } \\
\text { (Chin.) }\end{array}$ & $\begin{array}{l}\text { Rowing, } \\
\text { track } \\
\text { and } \\
\text { field, } \\
\text { swimming, } \\
\text { table } \\
\text { tennis }{ }^{(4)}\end{array}$ & $\begin{array}{c}15-26 \\
\left(\begin{array}{c}\text { mean } \\
20)\end{array}\right.\end{array}$ & 41 & $\begin{array}{l}\text { Exercise-induced } \\
\text { fatigue }\end{array}$ & N/A & & & $\begin{array}{l}\text { (1) Training- } \\
\text { related } \\
\text { condition } \\
\text { (2) Changes } \\
\text { level of } \\
\text { blood } \\
\text { testosterone } \\
\text { and } \\
\text { hemoglobin }\end{array}$ & $\begin{array}{l}\text { (1) Significantly } \\
\text { improved } \\
\text { (2) Significantly } \\
\text { increased }\end{array}$ & ND & (1), (2) N/A & $\begin{array}{c}\text { Catgut } \\
\text { implantation at } \\
\text { acupoints can } \\
\text { significantly } \\
\text { improve exercise } \\
\text { fatigue in the } \\
\text { player and the } \\
\text { mechanism is } \\
\text { possibly related } \\
\text { with increase of } \\
\text { testosterone and } \\
\text { hemoglobin levels. }\end{array}$ \\
\hline 16 & $\begin{array}{l}\text { Garlanger, } \\
2017 \\
{[29]}\end{array}$ & $\begin{array}{l}\text { USA } \\
\text { (Eng.) }\end{array}$ & $\underset{\text { ski }}{\text { Nordic }}$ & $14-17$ & 15 & $\begin{array}{c}\text { Exercise-related } \\
\text { DOMS and sense } \\
\text { of well-being }\end{array}$ & N/A & $\mathrm{N} / \mathrm{A}$ & $\begin{array}{l}\text { (1) MA + others } \\
\text { (2) } 2 \mathrm{wk} \\
\text { (3) } 1-5\end{array}$ & $\begin{array}{l}\text { (1) Time } \\
\text { required by } \\
\text { research } \\
\text { staff on } \\
\text { treatment } \\
\text { days } \\
\text { (2) Cost } \\
\text { (3) AEs } \\
\text { (4) } \\
\text { Participant/ } \\
\text { provider } \\
\text { ratio } \\
\text { (5) Effect on } \\
\text { DOMS } \\
\text { 6) Effect on } \\
\text { sense of } \\
\text { well-being }\end{array}$ & $\begin{array}{l}\text { (1) } 90 \mathrm{~min} \\
\text { (2) } 1500 \text { USD } \\
\text { (3) (See next } \\
\text { column) } \\
\text { (4) 7:1 } \\
\text { (5) significantly } \\
\text { improved ( } p< \\
0.05) \\
\text { 6) NS }\end{array}$ & $\begin{array}{l}\text { No } \\
\text { severe } \\
\text { AEs; } \\
\text { Minor } \\
\text { AEs } \\
\text { (73\%, } \\
\text { site pain, } \\
\text { etc.) }\end{array}$ & (1), (2) N/A & $\begin{array}{c}\text { Providing } \\
\text { acupuncture to } \\
\text { adolescent Nordic } \\
\text { ski athletes in the } \\
\text { practice field under } \\
\text { extreme } \\
\text { temperature is } \\
\text { feasible with the } \\
\text { appropriate } \\
\text { resources. }\end{array}$ \\
\hline 17 & $\begin{array}{l}\text { Luetmer, } \\
2019 \\
{[30]}\end{array}$ & $\begin{array}{l}\text { USA } \\
\text { (Eng.) }\end{array}$ & Football & $\begin{array}{c}13-18 \\
(\text { mean } \\
16)\end{array}$ & 42 & $\begin{array}{c}\text { Exercise-related } \\
\text { DOMS and sense } \\
\text { of well-being }\end{array}$ & $\mathrm{N} / \mathrm{A}$ & $\mathrm{N} / \mathrm{A}$ & $\begin{array}{c}\text { (1) MA + others } \\
\text { (2) } 5 \text {-day } \\
\text { (3) } 5(n=1(1) ; 4(n \\
=1(3) ; 3(n=8) ; 2 \\
(n=6) ; 1(n=(4)\end{array}$ & $\begin{array}{l}\text { Same as } \\
\text { above }\end{array}$ & $\begin{array}{l}\text { (1) } 75 \mathrm{~min} \\
\text { (2) } 700 \text { USD } \\
\text { (3) (See next } \\
\text { column) } \\
\text { (4) } 7-10: 1 \\
\text { (5) significantly } \\
\text { improved ( } p< \\
0.001) \\
\text { (6) NS }\end{array}$ & $\begin{array}{l}\text { No } \\
\text { severe } \\
\text { AEs; } \\
\text { Minor } \\
\text { AEs } \\
(55 \%, \\
\text { mild } \\
\text { focal } \\
\text { numbness } \\
\text { or } \\
\text { tingling) }\end{array}$ & (1), (2) N/A & $\begin{array}{l}\text { Effectively } \\
\text { providing } \\
\text { acupuncture to } \\
\text { multiple adolescent } \\
\text { football players in } \\
\text { their training } \\
\text { enviroment is } \\
\text { feasible with } \\
\text { appropriate staff } \\
\text { and resources. }\end{array}$ \\
\hline
\end{tabular}


Table 1. Cont.

\begin{tabular}{|c|c|c|c|c|c|c|c|c|c|c|c|c|c|c|}
\hline \multicolumn{3}{|c|}{ Study } & \multicolumn{3}{|c|}{ Demographic Information } & \multicolumn{2}{|c|}{$\begin{array}{l}\text { Main Concerns and Symptoms } \\
\text { of the Patient }\end{array}$} & \multirow[b]{2}{*}{$\begin{array}{c}\text { Medical History } \\
\\
\text { Oral/topical } \\
\text { NSAIDs, muscle } \\
\text { relaxant, } \\
\text { physiotherapy, } \\
\text { immobilization } \\
(7)\end{array}$} & \multirow{2}{*}{$\begin{array}{c}\begin{array}{c}\text { Administration } \\
\text { of Intervention }\end{array} \\
\\
\\
\text { (1) } \mathrm{MA}+\text { others } \\
\text { (2) } 6 \mathrm{wk}(2 / \mathrm{wk}) \\
\text { (3) } 12\end{array}$} & \multicolumn{5}{|c|}{ Follow-up and Outcomes } \\
\hline 18 & $\begin{array}{l}\text { Abe, } \\
2014 \\
{[48]}\end{array}$ & $\begin{array}{l}\text { Brazil } \\
\text { (Eng.) }\end{array}$ & $\begin{array}{l}\text { Various } \\
\text { sports } \\
\text { (Paraly } \\
\text { mpic } \\
\text { athletes) } \\
\text { (5) }\end{array}$ & $\underset{35}{\operatorname{Mean}}=$ & 7 & $\begin{array}{l}\text { Persistent pain } \\
\text { (shoulder, elbow, } \\
\text { spine, or knee) }{ }^{(6)}\end{array}$ & $\begin{array}{c}\text { \#1: 4-wk; } \\
\text { \#2-\#4, \#6: } \\
\text { chronic, } \\
\text { intermittent; } \\
\text { \#5: 2-wk; \#\#: } \\
\text { 8-wk }\end{array}$ & & & $\begin{array}{l}\text { (1) Pain } \\
\text { (VAS) } \\
\text { (2) Pain } \\
\text { (MPQ) }\end{array}$ & $\begin{array}{l}\text { (1), (2) Significant } \\
\text { pain reduction }\end{array}$ & $\begin{array}{c}\text { No } \\
\text { severe } \\
\text { AEs; } \\
\text { Minor } \\
\text { AEs } \\
\text { (episodic } \\
\text { local } \\
\text { bleeding); } \\
\text { One } \\
\text { athlete } \\
\text { died of } \\
\text { liver } \\
\text { cancer } \\
\text { after } \\
\text { this } \\
\text { study }\end{array}$ & (1), (2) ND & $\begin{array}{l}\text { Pain symptoms } \\
\text { were reduced with } \\
\text { acupuncture. The } \\
\text { mean duration } \\
\text { required for } \\
\text { improvement was } \\
\text { eight acupuncture } \\
\text { sessions. }\end{array}$ \\
\hline 19 & $\begin{array}{l}\text { Lin, } \\
2016 \\
{[28]}\end{array}$ & $\begin{array}{l}\text { USA } \\
\text { (Eng.) }\end{array}$ & $\begin{array}{l}\text { \#1: Ice } \\
\text { hockey } \\
\text { \#2: Field } \\
\text { hockey } \\
\text { and } \\
\text { lacrosse } \\
\text { \#3: } \\
\text { Soccer }\end{array}$ & $\begin{array}{l}\# 1: 8 ; \\
\# 2: 15 ; \\
\# 3: 18\end{array}$ & 3 & $\begin{array}{l}\text { Sport-related } \\
\text { postconcussive } \\
\text { symptoms }\end{array}$ & $\begin{array}{l}\# 1, \# 2: 1 \\
\text { mon; \#3: } \\
1.5 \text { mon }\end{array}$ & $\begin{array}{l}\text { Physical therapy, } \\
\text { medications }{ }^{(8)}\end{array}$ & $\begin{array}{c}\text { (1) MA + EA + } \\
\text { others } \\
\text { (2) \#1, \#2, 6-wk; } \\
\# 3,14-w k(1 / w k) \\
\text { (3) \#1, \#2, 6; \#3, } 14\end{array}$ & $\begin{array}{l}\text { (1) Pain } \\
\text { (VAS) } \\
\text { (2) Pain } \\
\text { (BPI) } \\
\text { (3) } \\
\text { Postconcussive } \\
\text { symptoms } \\
\text { questionnaire }\end{array}$ & $\begin{array}{c}(1)-(3) \\
\text { Satisfactory } \\
\text { symptomatic } \\
\text { reduction in all } \\
\text { patients }\end{array}$ & None & $\begin{array}{l}\text { (1) \#1, \#2: ND; \#3: } \\
\text { Return to all } \\
\text { sports activities } \\
\text { (All patients } \\
\text { returned to their } \\
\text { physical, social, } \\
\text { and school } \\
\text { activities) } \\
\text { (2) ND }\end{array}$ & $\begin{array}{l}\text { Acupuncture with } \\
\text { conventional } \\
\text { medication } \\
\text { appeared to reduce } \\
\text { the postconcussive } \\
\text { symptoms in the } 3 \\
\text { patients. }\end{array}$ \\
\hline 20 & $\begin{array}{l}\text { Yuill, } \\
2012 \\
{[44]}\end{array}$ & $\begin{array}{l}\text { Canada } \\
\text { (Eng.) }\end{array}$ & $\begin{array}{l}\# 1: \\
\text { Soccer } \\
\# 2, \# 3: \\
\text { Soccer } \\
\text { and } \\
\text { hockey }\end{array}$ & $\begin{array}{l}\# 1: 23 ; \\
\text { \#2: } 18 ; \\
\# 3: 29\end{array}$ & 3 & $\begin{array}{l}\text { Sports hernia } \\
\text { (chronic groin } \\
\text { pain) }\end{array}$ & $\begin{array}{c}\text { \#1: } 5 \text { wk; \#2: } \\
\text { 3-mon; \#3: } \\
\text { 5-wk }\end{array}$ & $\begin{array}{l}\text { \#1: None; \#2: Ice, } \\
\text { interferential } \\
\text { current, } \\
\text { ultrasound (2 } \\
\text { mon); \#3: } \\
\text { Physiotherapy, } \\
\text { adductor } \\
\text { stretching/ } \\
\text { strengthening, } \\
\text { interferential } \\
\text { current, } \\
\text { ultrasound }\end{array}$ & $\begin{array}{l}\text { (1) MA + EA + } \\
\text { others } \\
\text { (2) 6-8-wk } \\
\text { (1-2/wk) } \\
\text { (3) 6-16 }\end{array}$ & $\begin{array}{l}\text { (1) Pain } \\
\text { (VAS) } \\
\text { (2) Resisted } \\
\text { muscle } \\
\text { testing }\end{array}$ & $\begin{array}{l}\text { (1) 0/10 in all } \\
\text { cases at the time } \\
\text { of patient } \\
\text { discharge } \\
\text { (2) All positive } \\
\text { tests changed to } \\
\text { negative }\end{array}$ & ND & $\begin{array}{l}\text { (1) Within 3-4 } \\
\text { days of last } \\
\text { therapy } \\
\text { (2) None ( } 2 \mathrm{yr})\end{array}$ & $\begin{array}{l}\text { Three soccer } \\
\text { players, of varying } \\
\text { levels of ability, } \\
\text { presenting with a } \\
\text { suspected sports } \\
\text { hernia were relieved } \\
\text { of their pain after } 8 \\
\text { weeks of } \\
\text { conservative care. }\end{array}$ \\
\hline 21 & $\begin{array}{l}\text { Donoyama, } \\
2011 \\
{[43]}\end{array}$ & $\begin{array}{l}\text { Japan } \\
\text { (Eng.) }\end{array}$ & $\begin{array}{l}\text { Middle- } \\
\text { and } \\
\text { long- } \\
\text { distance } \\
\text { running }\end{array}$ & 26 & 1 & Amenorrhoea & Several mon & $\begin{array}{c}\text { A } \\
\text { norgestrel-ethinyl } \\
\text { estradiol } \\
\text { combination } \\
\text { (2-wk] }\end{array}$ & $\begin{array}{l}\text { (1) MA + others } \\
\text { (2) } 13 \text { mon } \\
\text { (3) } 46\end{array}$ & $\begin{array}{l}\text { (1) } \\
\text { Menstrual } \\
\text { blood flow } \\
\text { (2) Basal } \\
\text { Body } \\
\text { Temperature } \\
\text { (3) Body } \\
\text { Weight } \\
\text { (4) Body Fat } \\
\text { Rate } \\
\text { (5) BMI }\end{array}$ & $\begin{array}{l}\text { (1) Amount and } \\
\text { duration of } \\
\text { menstrual flow } \\
\text { increased at } \\
\text { regular intervals } \\
\text { (2) Moved close } \\
\text { to the biphasic } \\
\text { pattern } \\
\text { (3)-(5) NS }\end{array}$ & $\mathrm{ND}$ & (1), (2) ND & $\begin{array}{l}\text { Acupuncture } \\
\text { treatment may be a } \\
\text { feasible treatment } \\
\text { option in the field } \\
\text { of sports medicine } \\
\text { to help competitive } \\
\text { athletes with } \\
\text { amenorrhoea. }\end{array}$ \\
\hline
\end{tabular}


Table 1. Cont.

\begin{tabular}{|c|c|c|c|c|c|c|c|c|c|c|c|c|}
\hline \multicolumn{3}{|c|}{ Study } & \multicolumn{2}{|c|}{ Demographic Information } & \multirow{2}{*}{$\begin{array}{c}\begin{array}{c}\text { Main Concerns and Symptoms } \\
\text { of the Patient }\end{array} \\
\begin{array}{c}\text { Yips } \quad 2 \mathrm{yr} \\
\end{array}\end{array}$} & \multirow{2}{*}{$\begin{array}{c}\text { Medical History } \\
\text { None }\end{array}$} & \multirow{2}{*}{$\begin{array}{c}\text { Administration } \\
\text { of Intervention } \\
\text { (1) MA } \\
\text { (2 Few weeks } \\
(1 / \mathrm{wk}+1 / 2-\mathrm{wk}) \\
\text { (3) } 8\end{array}$} & \multicolumn{5}{|c|}{ Follow-up and Outcomes } \\
\hline 22 & $\begin{array}{l}\text { Rosted, } \\
2005 \\
{[37]}\end{array}$ & $\begin{array}{c}\text { UK } \\
\text { (Eng.) }\end{array}$ & Golf & 65 & & & & Symptoms & $\begin{array}{c}\text { Symptoms } \\
\text { disappeared after } \\
1 \text { treatment }\end{array}$ & ND & $\begin{array}{l}\text { (1) Playing golf } \\
\text { using his right } \\
\text { hand without } \\
\text { symptoms } \\
\text { (2) None (2 yr) }\end{array}$ & $\begin{array}{l}\text { Acupuncture may } \\
\text { be worth trying in } \\
\text { patients with the } \\
\text { yips since this } \\
\text { condition is } \\
\text { otherwise difficult } \\
\text { to treat. }\end{array}$ \\
\hline \multicolumn{13}{|c|}{ 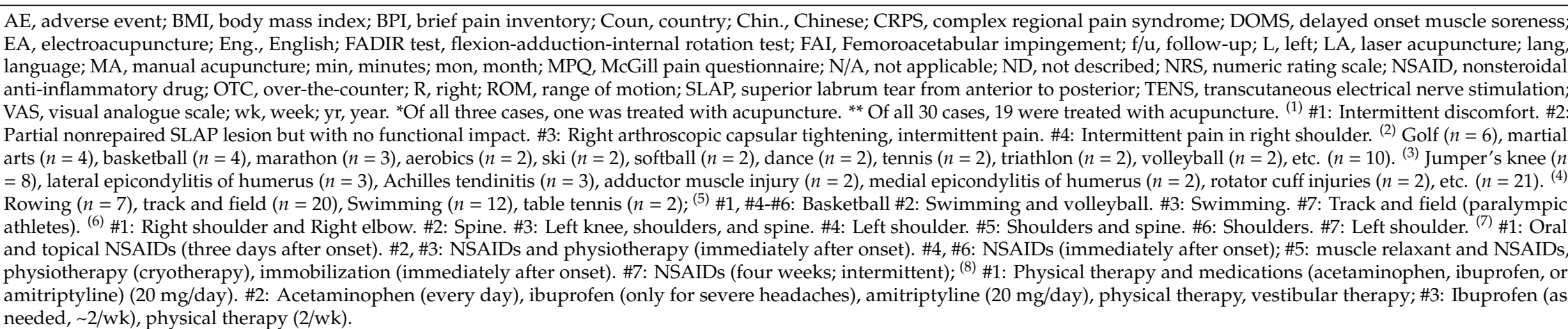 } \\
\hline
\end{tabular}


From 1980 to 2019, the number of relevant studies increased steadily with one study in the 1980s, two in the 1990s, six in the 2000s, and 13 in the 2010s. (Figure 2a). The studies were conducted in eight countries, with the largest number of studies in the United States $(n=7,31.8 \%)$; followed by Canada $(n=4)$; the UK $(n=3)$; China, Israel, and Japan $(n=2$, respectively); and Brazil and Puerto Rico $(n=1$, respectively) (Figure 2b). Two studies conducted in China [40,41] were written in Chinese and the rest were written in English.

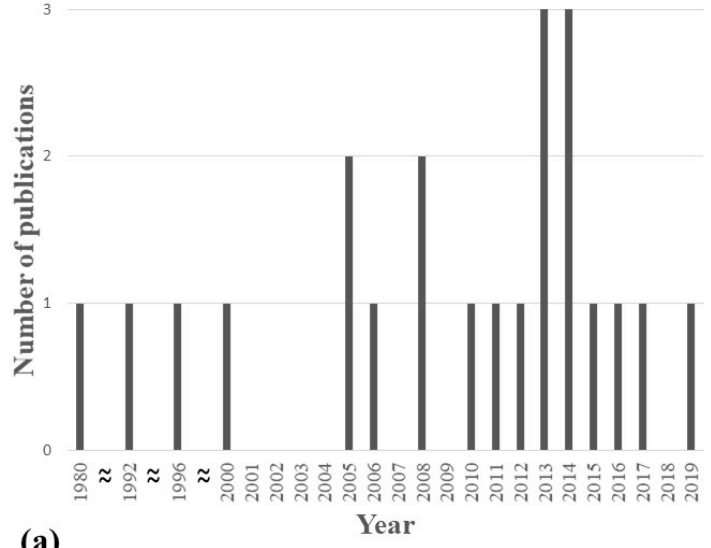

(a)

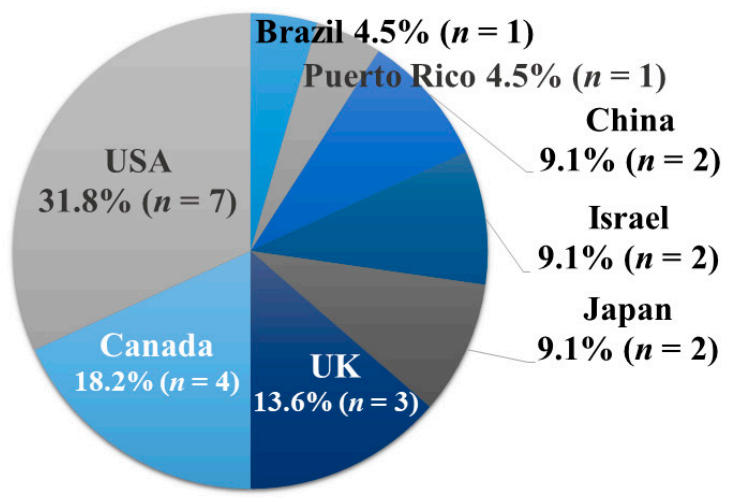

(b)

Figure 2. Characteristics of the included studies. (a) Number of published case reports/series by year; (b) frequency by country in which case reports/series were performed.

\subsection{Demographic Information, Main Concerns, and Symptoms of the Patient}

All subjects included in this review were athletes, and sports injuries were reported in various sports such as basketball ( $n=4,9$ cases); running, skiing, or volleyball $(n=3$, respectively); and field hockey, golf, soccer, football, ice hockey, swimming, or track and field ( $n=2$, respectively). The details of each sport are shown in Figure 3. Most studies $(n=14)$ reported cases for one-sport type of athlete, but five studies included athletes of various types of sports, and three studies did not report any specific sport types.

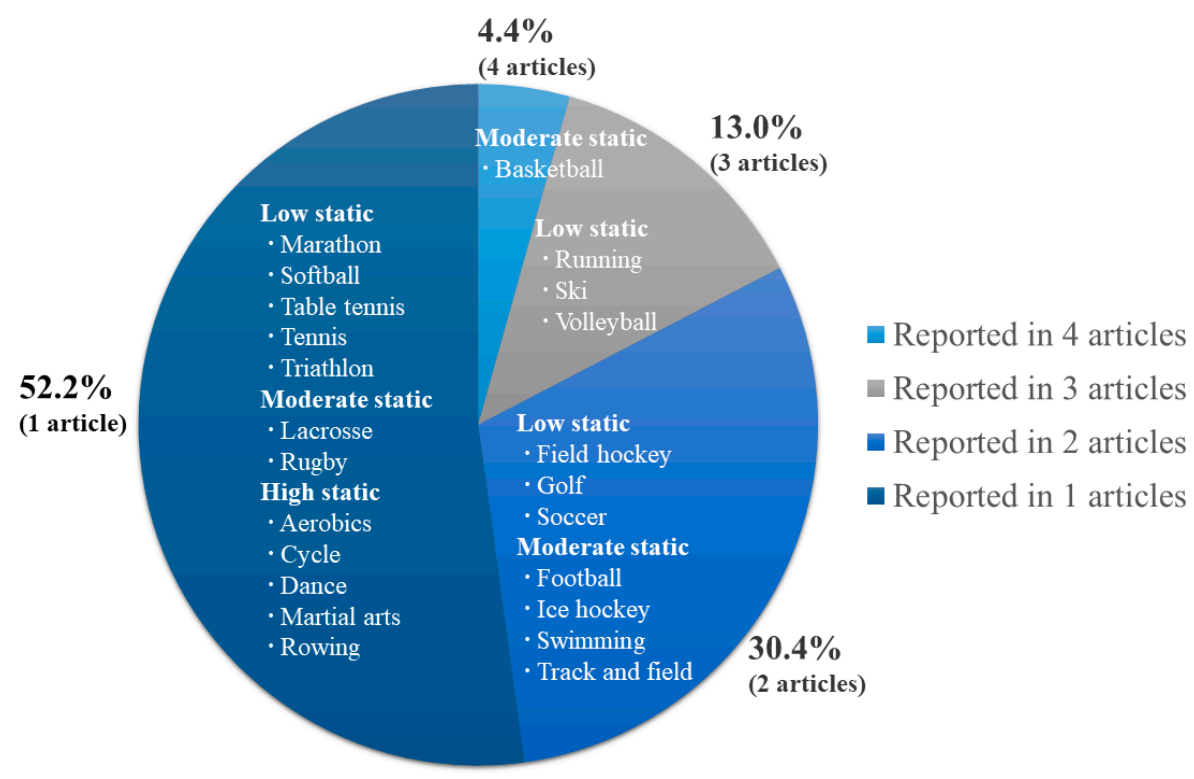

Figure 3. Type of sports. This figure shows how many case reports/series were performed according to the type of sports. For example, there were four case reports/series for basketball players, and three case reports/series for runners, skiers, and volleyball players, respectively. Each type of sports was classified and described according to the static components during competition. 
The total number of athletes included in our review was 211, of which $119(56.4 \%)$ were men. The athletes' ages ranged from 8 to 77 years old (mean $=24.8$ ).

Figure 4 shows the main concerns and symptoms of athletes in the included studies. Of the 211 cases of sports injuries, musculoskeletal system and connective tissue diseases accounted for the highest proportion of primary symptoms reported in each case report/series (98 cases, $n=14(46.4 \%)$ ). The most injured body regions were the knees (medial collateral ligament injury (19 cases), jumper's knee (8 cases), lateral meniscus injury (1 case)), followed by the elbows (lateral epicondylitis (4 cases), medial epicondylitis ( 2 cases), tendinopathy ( 1 case)), and shoulders (pain (4 cases), rotator cuff injury (2 cases)). In addition, studies of diverse musculoskeletal disorders were reported, including musculotendinous syndrome with chronic pain in multiple body regions (22 cases), complex regional pain syndrome, exertional rhabdomyolysis, and ganglion cysts of the foot (1 case, respectively) (Figure 5). On the other hand, sports injuries other than the musculoskeletal system were as follows. There were two studies [29,30] on exercise-related delayed onset muscle soreness (DOMS, 57 cases) and one study [40] on exercise-induced fatigue (41 cases) in athletes. Two studies reported mental and behavioral disorders such as yips for golfers (1 case) [37] and postconcussive symptoms (3 cases) [28]. Other studies included persistent pain in para-athletes ( 7 cases) [48], sports hernias ( 3 cases) [44], and amenorrhea ( 1 case) [43].

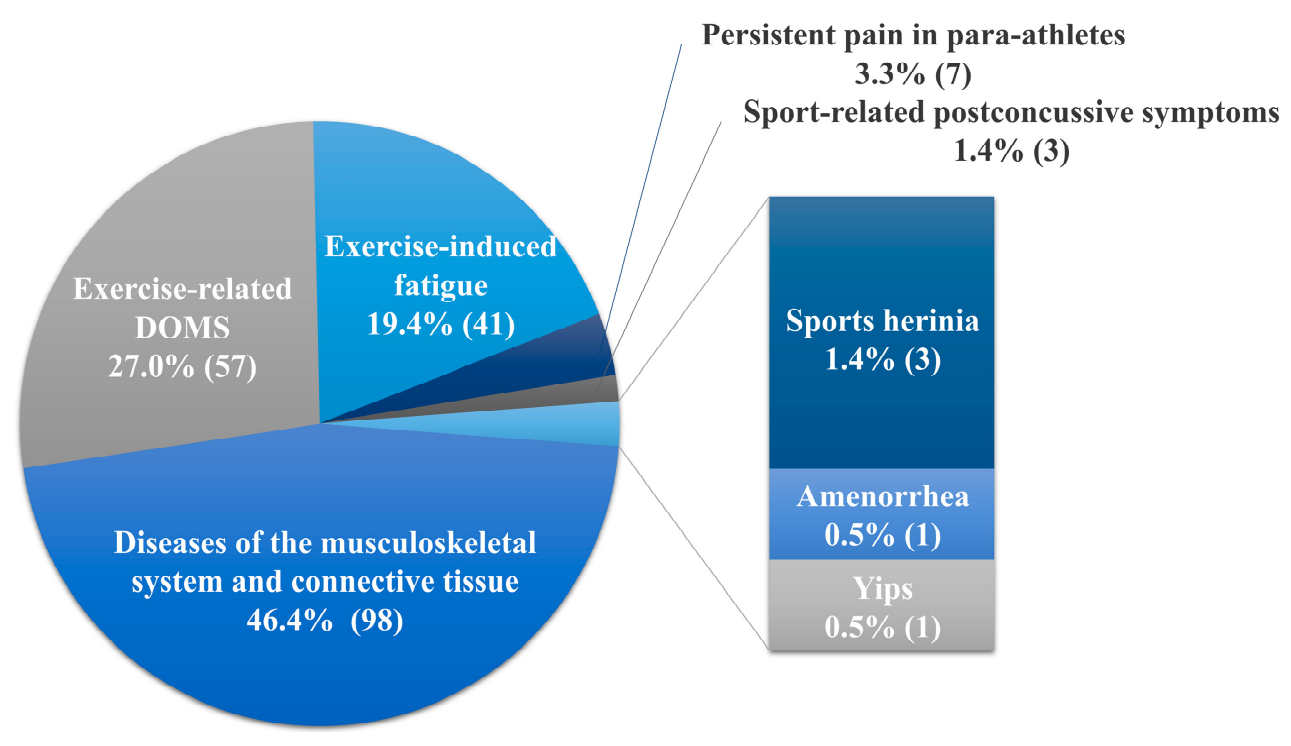

Figure 4. Main concerns and symptoms of the patients. 


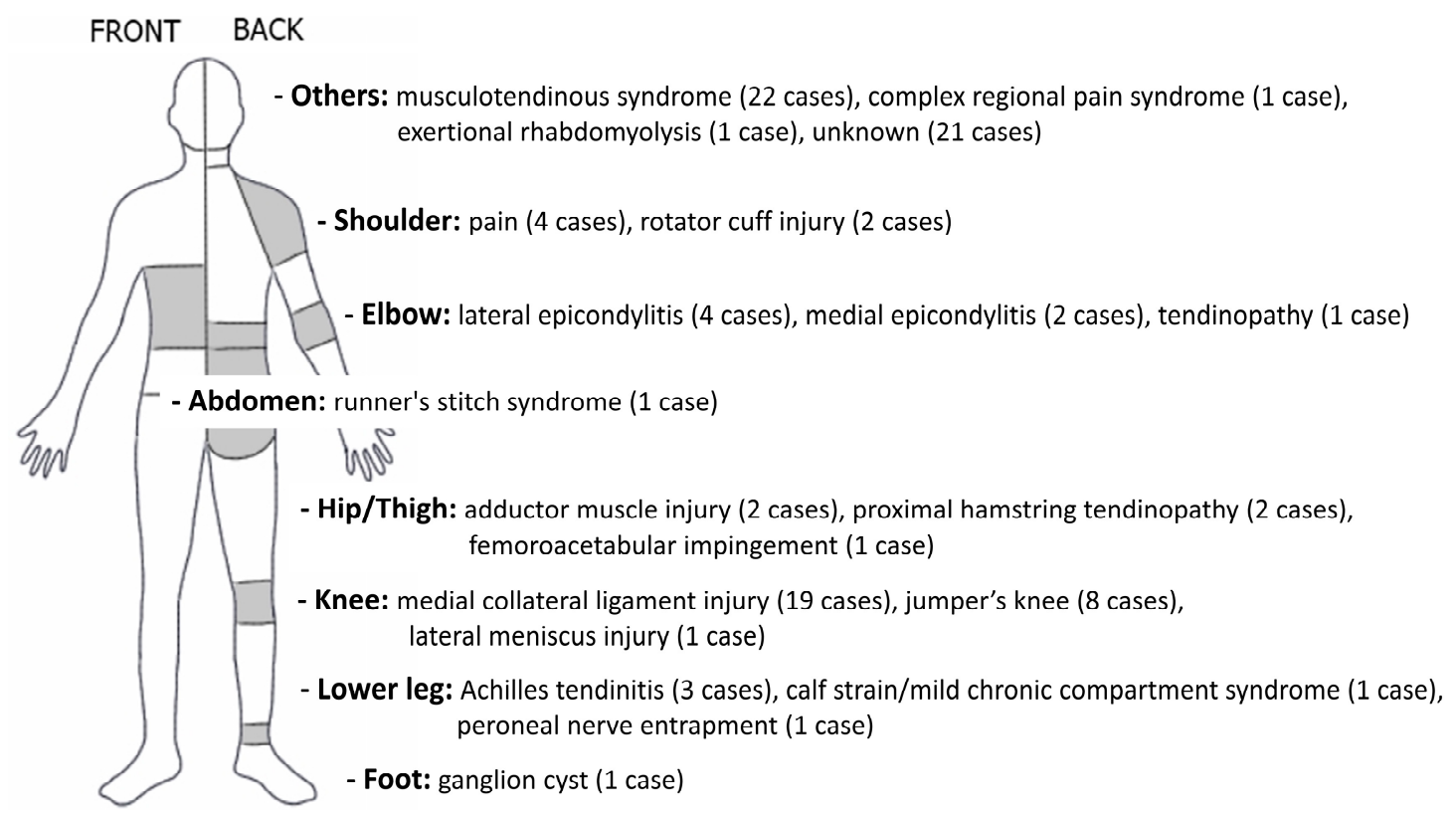

Figure 5. Injured body regions of musculoskeletal disease.

The duration of the athletes' disorder or symptoms varied from the day of onset to four years. According to relevant past interventions in each case report/series, among the patients of the included cases, except for seven studies, three cases had no past intervention, and all the other patients received conservative treatment such as physical therapy or medications for the symptoms. Of the seven studies excluded above, three studies conducted simply to observe the changes during the training periods did not require records for past intervention. The remaining four studies did not report relevant past interventions. The details are described in Table 1.

\subsection{Therapeutic Intervention}

\subsubsection{Acupuncture}

Twelve of the 22 studies included in our review reported the use of MA at acupoints or myofascial trigger points (MTrPs). For EA, four studies used EA alone, while three studies used MA with EA. Other studies demonstrated catgut embedding, TENS at acupoints or MTrPs, or low-level LA at acupoints or ashi points (acupuncture site, not given a specific name or definite location, determined by tenderness or other pathological reactions).

Some trends were observed between the types of acupuncture and the category of diseases utilized. For example, MA with acupoints was used in various symptoms in our review, such as yips, amenorrhea, or sports hernia, in addition to musculoskeletal disorders. On the other hand, studies in which MA was applied only to MTrP, study that used EA alone without MA, TENS study, and LA study were mainly used for athletes with diseases of the musculoskeletal system and connective tissue.

The treatment sites were reported in detail as specific acupoints in 15 studies (Table 2). Among the remaining seven studies, six described treatment sites, not as particular acupoints, but as muscles or ranges. In addition, one study did not describe any treatment sites. 
Table 2. Details of acupuncture and co-intervention.

\begin{tabular}{|c|c|c|c|c|c|}
\hline No. & Study ID & Sports Type & Disorders & $\begin{array}{l}\text { Details of Acupuncture } \\
\text { (Acupuncture Sites) }\end{array}$ & Co-Intervention \\
\hline \multicolumn{6}{|c|}{ Diseases of the musculoskeletal system and connective tissue } \\
\hline 1 & Dlin, 1980 [33] & ND & $\begin{array}{l}\text { Musculotendinous } \\
\text { syndromes of the chronic } \\
\text { exertion type (shoulder, } \\
\text { knee, anterior tibia, and } \\
\text { back pain) }\end{array}$ & $\begin{array}{l}\text { TENS (MTrPs } \\
\text { or acupoints) }\end{array}$ & None \\
\hline 2 & Sternfeld, $1992[34]$ & Long-distance running & Runner's stitch syndrome & $\begin{array}{l}\text { MA (PC3, LR14, ST36, } \\
\text { SP9, LR2, SP3) }\end{array}$ & None \\
\hline 3 & Seplow, 1996 [35] & Basketball & $\begin{array}{l}\text { Lateral meniscus injury (a } \\
\text { tear of the posterior horn } \\
\text { of the lateral meniscus) }\end{array}$ & $\begin{array}{l}\text { EA (ST41, GB43, } \\
\text { BL54, BL60) }\end{array}$ & $\begin{array}{l}\text { Ice, kinesiology, } \\
\text { resistance bands }\end{array}$ \\
\hline 4 & Hoven, $2000[36]$ & Ski & $\begin{array}{l}\text { Peroneal nerve } \\
\text { entrapment and } \\
\text { ankle sprain }\end{array}$ & $\begin{array}{l}\text { MA (ST40, GB34, } \\
\text { GB35, GB36) }\end{array}$ & $\begin{array}{c}\text { Chiropractic } \\
\text { manipulation (lumbar } \\
\text { spine, pelvis, lower } \\
\text { extremity), interferential } \\
\text { current, cryotherapy, soft } \\
\text { tissue mobilization }\end{array}$ \\
\hline 5 & Young, 2005 [38] & Rugby & $\begin{array}{l}\text { \#1: Calf strain and mild } \\
\text { chronic compartment } \\
\text { syndrome } \\
\text { \#2: Tennis elbow and } \\
\text { muscle tightness }\end{array}$ & $\begin{array}{c}\text { \#1: - EA (BL55-BL57, } \\
\text { BL57-GB35) } \\
\text { - MA (BL40, BL57, BL58, } \\
\text { BL60, BL65, GB34, } \\
\text { GB41, LI4) } \\
\text { \#2: - EA (ashi points, } \\
\text { EX-B2 from C3 to L5) } \\
\text { - MA (ashi points, GB21, } \\
\text { LI15, TE14, TE5, LI10, } \\
\text { LI11, LI12, LI5, LI4, } \\
\text { LI3, TE3) }\end{array}$ & $\begin{array}{l}\text { \#1: Tuina (gun-fa, rolling } \\
\text { technique; rou-fa, } \\
\text { kneading; plucking } \\
\text { (frictions)), stretching, } \\
\text { patient education } \\
\text { \#2: Tuina (gun-fa), } \\
\text { cupping (epicondyle) }\end{array}$ \\
\hline 6 & $\begin{array}{l}\text { Martínez-Silvestrini, } \\
2006 \text { [39] }\end{array}$ & ND & $\begin{array}{l}\text { CRPS after lower } \\
\text { extremity trauma and } \\
\text { clinical depression }\end{array}$ & $\begin{array}{l}\text { (for clinical depression } \\
\text { and pain disorder } \\
\text { treatment) } \\
\text { MA (ND) }\end{array}$ & $\begin{array}{l}\text { (for clinical depression } \\
\text { and pain } \\
\text { disorder treatment) } \\
\text { Fluoxetine (SSRIs) }\end{array}$ \\
\hline
\end{tabular}


Table 2. Cont

\begin{tabular}{|c|c|c|c|c|c|}
\hline No. & Study ID & Sports Type & Disorders & $\begin{array}{l}\text { Details of Acupuncture } \\
\text { (Acupuncture Sites) }\end{array}$ & Co-Intervention \\
\hline 7 & Yan, 2008 [41] & Football & $\begin{array}{l}\text { Sports injury in knee joint } \\
\text { medial collateral ligament }\end{array}$ & $\begin{array}{l}\text { EA (EX-LE2, } \\
\text { EX-LE5, GB34) }\end{array}$ & $\begin{array}{c}\text { Massage, physiotherapy, } \\
\text { cold pack, electric } \\
\text { stimulation, external } \\
\text { preparation, surgery, } \\
\text { splint, rehabilitation } \\
\text { training (as needed) }\end{array}$ \\
\hline 8 & Osborne, 2010 [42] & Volleyball & $\begin{array}{l}\text { Anterior/anterolateral } \\
\text { shoulder pain }\end{array}$ & $\begin{array}{c}\text { MA (MTrPs: } \\
\text { scapulohumeral muscles) }\end{array}$ & $\begin{array}{l}\text { Soft tissue therapy around } \\
\text { the shoulder, post-training } \\
\text { icing strategies, exercises } \\
\text { and stretching }\end{array}$ \\
\hline 9 & Pearcey, 2013 [46] & ND & $\begin{array}{l}\text { Exertional } \\
\text { rhabdomyolysis }\end{array}$ & $\begin{array}{l}\text { MA (from shoulder } \\
\text { to hand) }\end{array}$ & $\begin{array}{l}\text { Intravenous hydration } \\
\text { with sodium bicarbonate } \\
\text { and blood work }\end{array}$ \\
\hline 10 & Woitzik, 2013 [47] & Cycle & Ganglion cyst of the foot & $\begin{array}{l}\text { EA (ST36, LR3, } 2 \text { needles } \\
\text { inserted into the cyst on } \\
\text { opposite sides stimulated } \\
\text { at } 5 \mathrm{~Hz} \text { ) }\end{array}$ & None \\
\hline 11 & Morimoto, 2013 [45] & Various sports & $\begin{array}{l}\text { Jumper's knee, lateral } \\
\text { epicondylitis of humerus, } \\
\text { Achilles tendinitis, } \\
\text { adductor muscle injury, } \\
\text { medial epicondylitis of } \\
\text { humerus, rotator cuff } \\
\text { injuries, etc }\end{array}$ & $\begin{array}{l}\text { LA (low level laser } \\
\text { therapy at points } \\
\text { of pain/or } \\
\text { acupuncture points) }\end{array}$ & $\begin{array}{l}\text { Medication (NSAIDs) or a } \\
\text { poultice (as needed) }\end{array}$ \\
\hline 12 & Gliedt, 2014 [49] & Basketball & $\begin{array}{l}\text { Sub-acute left elbow } \\
\text { tendinopathy }\end{array}$ & $\begin{array}{l}\text { MA (palpated areas of } \\
\text { tenderness, just distal to } \\
\text { the insertion site of the } \\
\text { common wrist } \\
\text { extensor muscles) }\end{array}$ & $\begin{array}{c}\text { HVLA spinal } \\
\text { manipulation (thoracic } \\
\text { spine), ART (elbow), } \\
\text { home exercise program } \\
\text { (Brugger's exercises for } \\
\text { postural dysfunction) }\end{array}$ \\
\hline 13 & Jayaseelan, 2014 [50] & Running & $\begin{array}{l}\text { Proximal hamstring } \\
\text { tendinopathy }\end{array}$ & $\begin{array}{l}\text { MA (MTrPs: } \\
\text { medial/lateral hamstrings, } \\
\text { adductor magnus muscle) }\end{array}$ & $\begin{array}{c}\text { Eccentric training of } \\
\text { hamstrings, lumbopelvic } \\
\text { stabilization exercises, } \\
\text { patient education }\end{array}$ \\
\hline
\end{tabular}


Table 2. Cont

\begin{tabular}{|c|c|c|c|c|c|}
\hline No. & Study ID & Sports Type & Disorders & $\begin{array}{l}\text { Details of Acupuncture } \\
\text { (Acupuncture Sites) }\end{array}$ & Co-Intervention \\
\hline 14 & MacIntyre, 2015 [27] & Ice hockey & FAI & $\begin{array}{c}\text { EA (2 Hz, L2-L5 } \\
\text { bilaterally, and hip } \\
\text { muscles and nerves, SP12, } \\
\text { LR10, GB29, BL53, BL54) }\end{array}$ & $\begin{array}{c}\text { Soft tissue therapy, spinal } \\
\text { manipulative therapy, } \\
\text { MWM and } \\
\text { rehabilitation exercises }\end{array}$ \\
\hline \multicolumn{6}{|c|}{ Exercise-induced fatigue } \\
\hline 15 & Chen, 2008 [40] & $\begin{array}{l}\text { Rowing, track and field, } \\
\text { swimming, table tennis }\end{array}$ & Exercise-induced fatigue & $\begin{array}{l}\text { Catgut embedding (CV4, } \\
\text { BL23, GV4, ST36, SP6 } \\
\text { and etc.) }\end{array}$ & None \\
\hline 16 & Garlanger, 2017 [29] & Nordic ski & $\begin{array}{l}\text { Exercise-related DOMS } \\
\text { and sense of well-being }\end{array}$ & $\begin{array}{l}\text { MA (when underlying } \\
\text { muscle soreness occurred: } \\
\text { SP10, ST34, SP9, ST36, } \\
\text { GB34, BL56; if no specific } \\
\text { muscle soreness: ST36, } \\
\text { GB34, ST34) }\end{array}$ & $\begin{array}{l}\text { NSAIDs }(24-48 \mathrm{~h} \text { prior to } \\
\text { the study, } n=6 \text {; between } \\
\text { treatment days } 2 \text { and } 3, \\
n=3 \text {; between treatment } \\
\text { days } 4 \text { and } 5, n=1)\end{array}$ \\
\hline 17 & Luetmer, 2019 [30] & Football & $\begin{array}{l}\text { Exercise-related DOMS } \\
\text { and sense of well-being }\end{array}$ & $\begin{array}{l}\text { MA (when underlying } \\
\text { muscle soreness occurred: } \\
\text { SP10, ST34, SP9, ST36, } \\
\text { GB34, BL56; if no specific } \\
\text { muscle soreness: ST36, } \\
\text { GB34, ST34) }\end{array}$ & $\begin{array}{c}\text { NSAIDs or } \\
\text { acetaminophen (before } \\
\text { day } 1, n=7 \text {; between day } \\
1 \text { and } 2, n=7 ; \text { between } \\
\text { days } 2 \text { and } 3, n=10 ; \\
\text { between days } 3 \text { and } 4, \\
n=8 \text {; between days } 4 \\
\text { and } 5, n=9 \text { ) }\end{array}$ \\
\hline \multicolumn{6}{|l|}{ Others } \\
\hline 18 & Abe, 2014 [48] & $\begin{array}{c}\text { Various sports } \\
\text { (Paralympic athletes) }\end{array}$ & $\begin{array}{l}\text { Persistent pain (shoulder, } \\
\text { elbow, spine, or knee) }\end{array}$ & $\begin{array}{c}\text { MA (CV12, CV3, LI4, LU7, } \\
\text { HT7, SP10, ST36, } \\
\text { LR2, KI3) }\end{array}$ & $\begin{array}{c}\text { The training schedule and } \\
\text { the usual treatment } \\
\text { regimens maintained }\end{array}$ \\
\hline 19 & Lin, 2016 [28] & $\begin{array}{l}\text { Ice hockey, field hockey, } \\
\text { lacrosse, soccer }\end{array}$ & $\begin{array}{c}\text { Sport-related } \\
\text { postconcussive symptoms }\end{array}$ & $\begin{array}{l}\text { - MA (\#1: LI4, ST36, LR3, } \\
\text { TE5, Ashi points; \#2: LI4, } \\
\text { ST36, LR3, TE5, SP6, } \\
\text { Yin-Tang, Ashi points; \#3: } \\
\text { LI4, ST36, SP6, LR3, TE23, } \\
\text { GV20, Yin-Tang, TE5, } \\
\text { LI11, ST7, BL4, BL6, TE9, } \\
\text { GB21, GB20, Ashi points) } \\
\text { - EA(2-Hz, ST 36) }\end{array}$ & $\begin{array}{l}\text { Cupping (Back Shu } \\
\text { points), Gua sha (Back } \\
\text { Shu points, treatment } \\
\text { involving repeated } \\
\text { pressured strokes over } \\
\text { body surface with a } \\
\text { smooth edged tool), } \\
\text { conventional medical } \\
\text { therapies maintained }\end{array}$ \\
\hline
\end{tabular}


Table 2. Cont.

\begin{tabular}{|c|c|c|c|c|c|}
\hline No. & Study ID & Sports Type & Disorders & $\begin{array}{c}\text { Details of Acupuncture } \\
\text { (Acupuncture Sites) }\end{array}$ & Co-Intervention \\
\hline 20 & Yuill, 2012 [44] & Soccer, hockey & $\begin{array}{l}\text { Sports hernia (chronic } \\
\text { groin pain) }\end{array}$ & $\begin{array}{c}\text { - EA (2 Hz, L2-L4, } \\
\text { T10-T12 bilaterally) } \\
\text { - MA (\#1: SP 13, ST 29, } \\
\text { ST30, KI11, GB28 \#2: ST29, } \\
\text { ST30, LR9, LR10 \#3: } \\
\text { ST30, LR10) }\end{array}$ & $\begin{array}{l}\text { Soft tissue therapy, laser } \\
\text { therapy and microcurrent } \\
\text { (site of the chief } \\
\text { complaint), stretching, } \\
\text { Wobenzyme (2400mg tabs, } \\
\text { 2/day, } 2 \text { weeks), } \\
\text { rehabilitation therapy, } \\
\text { plyometric training } \\
\text { (3/week, } 8 \text { weeks) }\end{array}$ \\
\hline 21 & Donoyama, 2011 [43] & $\begin{array}{l}\text { Middle- and long- } \\
\text { distance running }\end{array}$ & Amenorrhoea & $\begin{array}{l}\text { MA (CV6, CV12, LR3, } \\
\text { LR14, BL17, BL18, BL23, } \\
\text { SP6, SP10) }\end{array}$ & $\begin{array}{l}\text { A norgestrel-ethinyl } \\
\text { estradiol combination } \\
\text { (as needed) }\end{array}$ \\
\hline 22 & Rosted, 2005 [37] & Golf & Yips & $\begin{array}{c}\text { MA (GV20, } \\
\text { EX-HN-1, TE5) }\end{array}$ & None \\
\hline
\end{tabular}

ART, active release techniques; BL, bladder meridian; CRPS, Complex regional pain syndrome; CV, conception vessel meridian; DOMS, Delayed onset muscle soreness; EA, electroacupuncture; EX-B, extra point (back); EX-HN, extra point (head and neck); EX-LE, extra point (lower extremity); FAI, Femoroacetabular impingement; GB, gallbladder meridian; GV, governor vessel meridian; HT, heart meridian; HVLA, high-velocity low-amplitude; KI, kidney meridian; LA, laser acupuncture; LI, large intestine meridian; LR, liver meridian; LU, lung meridian; MA, manual acupuncture; MTrPs, myofascial trigger points; MWM, Mulligan Mobilizations with Movement; ND, not described; NSAID, nonsteroidal anti-inflammatory drug; PC, pericardium meridian; SP, spleen meridian; SSRIs, selective serotonin reuptake inhibitors; ST, stomach meridian; TE, triple energizer meridian; TENS, transcutaneous electrical nerve stimulation. 


\subsubsection{Other Intervention (Co-Intervention)}

In 12 studies that applied MA, only two studies that described golfer's yips and runner's stitch syndrome used MA alone, and all other studies used a variety of co-interventions with acupuncture. Moreover, excluding three studies applying TENS for musculotendinous syndromes, catgut embedding for exercise-induced fatigue, or EA for ganglion cysts of the foot, the other 10 studies used a combination of co-interventions with acupuncture. The types of co-intervention differed depending on the disease; most commonly used were medication, Tuina, and various physical therapies (Table 2).

\subsubsection{Treatment Duration and Session}

The duration of treatment and follow-up periods for patients in each study varied from a few days to 13 months. With the exception of four studies that did not report the number of treatments, the study that provided the largest number of acupuncture treatments was the amenorrhea study in female runners (46 acupuncture treatment sessions over 13 months). Most of the others showed fewer than 10 treatment sessions.

Of the 11 studies reporting treatment frequency, the most common was a once- or twice-a-week regimen. Administration of treatment once a day or once every two weeks was reported in only one study, respectively.

Two studies showed that acupuncture was used only for some of the treatment periods: one study [50] used acupuncture in the second phase of the total three-phase treatment course, and the other study [35] used acupuncture only during the first two weeks.

\subsection{Follow-Up and Outcomes}

\subsubsection{Outcome Measures}

The outcome measures used in the studies can be categorized as follows: (1) Subjective outcome measures, (2) objective outcome measures, (3) AEs, (4) information on RTP, and (5) recurrence rates, including follow-up results.

Many studies described narratively regarding the alteration or improvement of patients' symptoms $(n=9)$. Different types of pain scales were used for an array of diseases that involve pain, including those of the musculoskeletal system, as follows $(n=7)$ : Numerical rating scale (NRS), brief pain inventory (BPI), visual analog scale (VAS), pain relief score (PRS), McGill Pain Questionnaire (MPQ). There were also some objective outcome measures, such as blood test results, blood flow changes, body temperature, or numerical motor function evaluation outcome measures, including range of motion $(\mathrm{ROM})(n=6)$. Additionally, in some cases, results were comprehensively described on treatment days and cost for treatment, AEs, participants/providers ratio, effects on training, and recovery time, for the purpose of validity and feasibility evaluation of treatment intervention $[29,30]$.

\subsubsection{Results}

Most of the main outcomes focused on positive changes in patients. This can also be confirmed by the authors' comments within each study on acupuncture treatment (Table 1). Of the 13 studies on the diseases of the musculoskeletal system and connective tissue, three studies used acupuncture only as an adjunctive treatment and acupuncture was not mentioned in the authors' conclusions [39,41,46]. Several studies reported that MA played a significant role in the treatment of shoulder pain, elbow tendinopathy, proximal hamstring tendinopathy, or peroneal nerve entrapment. EA was highly recommended as a conservative intervention for the treatment of femoroacetabular impingement [27], lateral meniscus injury [35], and ganglion cysts of the foot [47]. According to Young [38], EA plus MA is very effective in the treatment of calf strain and tennis elbow. LA and TENS are effective in musculoskeletal disorders involving several regions.

In mental and behavioral disorders, MA for golfer's yips and MA plus EA for sports-related postconcussive symptoms appeared to significantly reduce the symptoms. MA is beneficial and feasible 
for treating exercise-related DOMS in adolescent athletes and persistent pain in para-athletes. Catgut implantation at acupoints for sports fatigue or MA plus EA on soccer players with sports hernias appeared to significantly alleviate symptoms. As a result of Donoyama's study [43], acupuncture or hormone replacement therapy for amenorrhea was not effective as a single intervention, but only when used in combination.

\subsubsection{Adverse Events}

AEs were reported in five studies (22.7\%), of which two reported no AEs, and the remaining three reported only minor AEs such as bleeding, pain, or numbness at the acupuncture site.

\subsubsection{Return to Play and Recurrence}

Results of the patients' RTP were reported in 12 studies (54.5\%). In contrast, relatively less follow-up data related to relapse were collected, with only seven studies (31.8\%) recording follow-up results to estimate recurrence rates (2-year follow-up period, $n=2$; less than 6 months follow-up period, $n=5$ ). As a result, only one study [33] reported that there was recurrence within the follow-up period; it was reported that six of 22 athletes treated for musculotendinous syndrome recurred between 10 days and two months after return to play.

\section{Discussion}

This systematic review summarized and analyzed case reports/series and series using acupuncture for sports injuries in athletes. Although the causal relationship of the effectiveness of acupuncture treatment was unclear due to the nature of the case report/series, it was confirmed that acupuncture was applied to a variety of sports injuries for athletes, ranging from yips of golf players to sports hernias of soccer players. The types of acupuncture also varied, including MA, EA, TENS, LA, and catgut embedding. The authors who performed each case report/series reported on the value of acupuncture as follows: (1) Short-term pain relief and recovery from dysfunction in each injured body region observed after acupuncture treatment could be beneficial in competitive elite athletes; (2) acupuncture has also been recognized for its clinical value as a useful noninvasive, conservative management for sports injuries such as lateral meniscus rupture, femoroacetabular impingement, ganglion cysts, and sports hernias; (3) acupuncture is worth trying for psychoneuromuscular impediments such as yips, which are poorly treated, and for exercise-related DOMS in athletes, and it has been suggested as an accessible treatment with appropriate resource (including cost and time).

Several previous RCTs have shown that acupuncture is effective in controlling pain in sports injuries in athletes, and it has been reported that the symptoms of rotator cuff tendinitis [51], shoulder impingement syndrome [52], shin splints [53], and plantar fasciitis [54] were improved by acupuncture. As of late, research on the effects and mechanisms of acupuncture tended to focus on pain control, but, clinically, it has been applied to various diseases and symptoms other than pain. Many studies related to pain control in acupuncture, which have been reported so far, have elucidated well the systemic and local mechanisms of acupuncture through mediators such as the central or autonomic nervous systems [19]. Nevertheless, research and discussion on the very complex elements involved in performing "acupuncture" are still in progress, and research on the specific mechanisms of the different diseases is still required.

As with the complexity of acupuncture, the scope of sports injuries is difficult and extensive to establish clearly. [55,56]. There are many different terms for sports injury, such as "sports impairment", "sports trauma", "sports incapacity", "sports disease", and "sports illness". If it can be recognized that each of these terms may have subtle differences in meaning, it can be seen that the need for a very complex perspective and a multidisciplinary approach in the field of sports medicine is inevitable. In fact, sports injury is known to require a systematic and multidisciplinary approach to treatment due to its complex etiology and various symptoms [7]. For example, overuse injuries in athletes are difficult to treat because symptoms are often extensive and uncharacteristic [57]. Another factor 
to consider in the treatment of sports injury in athletes is that they need not only recovery of the injured body part, but also recovery of their function to perform the sport again [15]. For athletes to return to play, systematic rehabilitation is necessary following successful treatment [58]. Physical and muscle strength, muscular endurance, flexibility, and agility need to be improved again as well as management of anxiety and depression caused by injury, if necessary [17]. Therefore, for sports injuries of elite athletes, an "RTP strategy" is required, and, for this, it has been discussed that the conservative method using a multimodal approach should be considered first [27]. However, apart from the lack of evidence of recommendations for various conservative treatment options [59], there are still limitations in the application of drug therapy, one of the most used conservative managements, to all cases of the RTP strategy [60]. The case studies included in this review suggest that acupuncture is one of the conservative approaches that would merit consideration in the field of sports and may be worth trying even for unexpected disorders that are not easy to treat [27-30,33-50].

Interestingly, case reports/series included in this review were mainly performed in the Americas and Europe, but not in the East Asian countries where acupuncture is primarily used in clinical practice. However, this does not denote that acupuncture treatments are not applied to sports injuries in East Asian countries. In Korea, acupuncture has been used formally or informally in sporting events since the early 1990s. In international sports competitions held in Korea that encompass a variety of sports, team doctors treated Korean athletes (sometimes including foreign athletes) with acupuncture and expressed satisfaction [61-63]. This positive experience has led to an opportunity for acupuncture to be introduced into the official medical treatment of the Olympic Village polyclinic in the 2018 PyeongChang Winter Olympics [64].

For a critical interpretation of this review, we suggest that careful consideration of the following limitations is essential. Firstly, the distinctive advantage of this review, but also an intricate disadvantage, was that we analyzed "sports injury" research on "athletes". Defining the terms "sports injury" and "athlete" involves a multitude of controversial concepts. [65] Thus, future research suggesting an accurate terminology will provide a clearer perspective. Second, it should be taken into account that the data we analyzed were derived from case reports/series. For our research to provide useful insights for clinical acupuncture for sports medicine practitioners, it is necessary to establish specific recommendations for which acupuncture is an effective treatment for the different types of sports injuries. This cannot be presented based only on case reports/series. The third limitation is linked to the second limitation. It should be noted that acupuncture treatment may not be a "study only to verify the effectiveness of acupuncture" in each case study. Since a number of studies included in this review used acupuncture as a co-intervention, or an adjunctive treatment, and not a stand-alone intervention, the effect of acupuncture can be a response to a multimodal conservative treatment. Fourth, our research showed that acupuncture can be applied to a variety of diseases within the sports injury category in athletes, but should not be misleading in the sense that acupuncture is effective for all variety of diseases. Experimental research such as RCTs for athletes, or systematic review based on it, can give a clear answer to the effectiveness of acupuncture. A recent systematic review of the effects of acupuncture treatment on DOMS reported that acupuncture had small-to-moderate effects compared to the no-treatment group and presented insufficient data for meta-analysis [66]. Further well-designed studies assessing acupuncture's efficacy in sports medicine are warranted to establish a strong evidence and to support clinical practice.

This study provided minor clues about the possibility of applying acupuncture to various types of sports injuries in athletes. In the future, we need to clarify which types of injuries are more effective in acupuncture through a detailed classification of sports injuries. As previously stated, it is very difficult to clearly identify the causes or symptoms of sports injuries. Nevertheless, in order to propose a more practical application of acupuncture, research that can generate evidence through more specific research questions and hypotheses should be conducted. 


\section{Conclusions}

In this review, we endeavored to identify the potential of acupuncture for managing discomfort in athletes and their return to exercise through case reports/series on sports injuries in athletes. The included cases showed some potential of acupuncture in the treatment of various types of sports injuries, beyond pain control in musculoskeletal disorders. In addition, this review will assist practitioners who wish to use acupuncture to treat sports injuries and enable practical clinical applications of acupuncture, thereby allowing the use of novel therapeutic options for patients. This is the first review, to our knowledge, to systematically search, summarize, and analyze clinical case reports/series on the potential application of acupuncture in the treatment of sports injuries of athletes.

Author Contributions: Conceptualization, S.-Y.K. and J.-H.L.; methodology, validation, formal analysis, investigation, and data curation, J.-W.L. and S.-Y.K.; resources, J.-H.L.; writing-original draft preparation, J.-W.L.; writing-review and editing, S.-Y.K. and J.-H.L.; visualization, J.-W.L. and S.-Y.K.; supervision, S.-Y.K.; project administration, S.-Y.K.; funding acquisition, J.-H.L. All authors have read and agreed to the published version of the manuscript.

Funding: This research was funded by the Korea Institute of Oriental Medicine (grant no. KSN 2013210) and supported by a grant of the Korea HealthTechnology R\&D Project through the Korea Health Industry Development Institute (KHIDI), funded by the Ministry of Health \& Welfare, Republic of Korea (grant number: HI17C1686).

Acknowledgments: A special thanks to Gyeong Hwan Hyeon (Gachon University) for helping in preparing the figures.

Conflicts of Interest: The authors declare no conflict of interest.

\section{References}

1. Warburton, D.E.R.; Bredin, S.S.D. Health benefits of physical activity: A systematic review of current systematic reviews. Curr. Opin. Cardiol. 2017, 32, 541-556. [CrossRef] [PubMed]

2. Pedersen, B.K.; Saltin, B. Exercise as medicine-Evidence for prescribing exercise as therapy in 26 different chronic diseases. Scand. J. Med. Sci. Sports 2015, 25, 1-72. [CrossRef] [PubMed]

3. Petridou, A.; Siopi, A.; Mougios, V. Exercise in the management of obesity. Metabolism 2019, 92, $163-169$. [CrossRef] [PubMed]

4. Parkkari, J.; Kujala, U.M.; Kannus, P. Is it possible to prevent sports injuries? Review of controlled clinical trials and recommendations for future work. Sports Med. 2001, 31, 985-995. [CrossRef] [PubMed]

5. Patel, D.R.; Baker, R.J. Musculoskeletal injuries in sports. Prim. Care Clin. Off Pract. 2006, 33, 545-579. [CrossRef] [PubMed]

6. Alessandrino, F.; Balconi, G. Complications of muscle injuries. J. Ultrasound 2013, 16, 215-222. [CrossRef] [PubMed]

7. Khan, K.; Brukner, P. Brukner \& Khan's Clinical Sports Medicine; McGraw-Hill Education: New York, NY, USA, 2011.

8. Šmela, P.; Pačesová, P.; Kraček, S.; Hájovský, D. Performance Motivation of Elite Athletes, Recreational Athletes and Non-Athletes. Acta Fac. Educ. Phys. Univ. Comen. 2017, 57, 125-133. [CrossRef]

9. Zippelius, K.; Schwarzinger, A. Sports injuries-improved healing processes due to integrative TCM therapy. Eur. J. Orient. Med. 2017, 8, 28-33.

10. Herring, S.A.; Kibler, W.B.; Putukian, M. The team physician and the return-to-play decision: A consensus statement-2012 update. Med. Sci. Sports Exerc. 2012, 44, 2446-2448. [CrossRef]

11. Ting, J.H.; Wallis, D.H. Medical management of the athlete: Evaluation and treatment of important issues in sports medicine. Clin. Podiatr. Med. Surg. 2007, 24, 127-158. [CrossRef]

12. Doyscher, R.; Kraus, K.; Finke, B.; Scheibel, M. Acute and overuse injuries of the shoulder in sports. Orthopade 2014, 43, 202-208. [CrossRef]

13. Krauss, A. Why all randomised controlled trials produce biased results. Ann. Med. 2018, 50, 312-322. [CrossRef]

14. Dainesi, S.M.; Goldbaum, M. Reasons behind the participation in biomedical research: A brief review. Rev. Bras. Epidemiol. 2014, 17, 842-851. [CrossRef] 
15. Grimes, D.A.; Schulz, K.F. Descriptive studies: What they can and cannot do. Lancet 2002, 359, $145-149$. [CrossRef]

16. Kuchar, E. The importance of case reports in current pediatric endocrinology and metabolism literature-The analysis of publications indexed in Medline in the years 2004-2009. Pediatr. Endocrinol. Diabetes Metab. 2010, 16, 119-122.

17. Gopikrishna, V. A report on case reports. J. Conserv. Dent. 2010, 13, 265-271. [CrossRef]

18. Yuan, Q.-L.; Wang, P.; Liu, L.; Sun, F.; Cai, Y.-S.; Wu, W.-T.; Ye, M.-L.; Ma, J.-T.; Xu, B.-B.; Zhang, Y.-G. Acupuncture for musculoskeletal pain: A meta-analysis and meta-regression of sham-controlled randomized clinical trials. Sci. Rep. 2016, 6, 30675. [CrossRef]

19. Filshie, J.; White, A.; Cummings, M. Medical Acupuncture: A Western Scientific Approach, 2nd ed.; Elsevier: Amsterdam, The Netherlands, 2016.

20. Han, J.-S. Acupuncture and endorphins. Neurosci. Lett. 2004, 361, 258-261. [CrossRef]

21. Cheng, K.J. Neurobiological Mechanisms of Acupuncture for Some Common Illnesses: A Clinician's Perspective. J. Acupunct. Meridian Stud. 2014, 7, 105-114. [CrossRef]

22. Pirnia, B.; Bazargan, N.M.; Hamdieh, M.; Pirnia, K.; Malekanmehr, P.; Maleki, F.; Zahiroddin, A. The Effectiveness of Auricular Acupuncture on the Levels of Cortisol in a Depressed Patient. Iran. J. Public Health 2019, 48, 1748-1750. [PubMed]

23. Hui, K.K.; Marina, O.; Liu, J.; Rosen, B.R.; Kwong, K.K. Acupuncture, the limbic system, and the anticorrelated networks of the brain. Auton. Neurosci. 2010, 157, 81-90. [CrossRef] [PubMed]

24. MacPherson, H.; Vickers, A.; Bland, M.; Torgerson, D.; Corbett, M.S.; Spackman, E.; Saramago, P.; Woods, B.; Weatherly, H.; Sculpher, M.; et al. Acupuncture for chronic pain and depression in primary care: A programme of research. Program. Grants Appl. Res. 2017, 5, 1-316. [CrossRef]

25. Cui, C.-L.; Wu, L.-Z.; Luo, F. Acupuncture for the Treatment of Drug Addiction. Neurochem. Res. 2008, 33, 2013-2022. [CrossRef]

26. Huang, Q.; Luo, D.; Chen, L.; Liang, F.X.; Chen, R. Effectiveness of Acupuncture for Alzheimer's Disease: An Updated Systematic Review and Meta-analysis. Curr. Med Sci. 2019, 39, 500-511. [CrossRef] [PubMed]

27. MacIntyre, K.; Gomes, B.; MacKenzie, S.; D’Angelo, K. Conservative management of an elite ice hockey goaltender with femoroacetabular impingement (FAI): A case report. J. Can. Chiropr. Assoc. 2015, 59, 398-409.

28. Lin, K.; Tung, C. Acupuncture for Recovery from Pediatric Sport-Related Concussion. Med Acupunct. 2016, 28, 217-222. [CrossRef]

29. Garlanger, K.L.; Fredericks, W.H.; Do, A.; Bauer, B.A.; Laskowski, E.R. The Feasibility and Effects of Acupuncture in an Adolescent Nordic Ski Population. PM R 2017, 9, 795-803. [CrossRef]

30. Luetmer, M.T.; Do, A.; Canzanello, N.C.; Bauer, B.A.; Laskowski, E.R. The Feasibility and Effects of Acupuncture on Muscle Soreness and Sense of Well-being in an Adolescent Football Population. Am. J. Phys. Med. Rehabil. 2019, 98, 964-970. [CrossRef]

31. Riley, D.S.; Barber, M.S.; Kienle, G.S.; Aronson, J.K.; von Schoen-Angerer, T.; Tugwell, P.; Kiene, H.; Helfand, M.; Altman, D.G.; Sox, H.; et al. CARE guidelines for case reports: Explanation and elaboration document. J. Clin. Epidemiol. 2017, 89, 218-235. [CrossRef]

32. Mitchell, J.H.; Haskell, W.L.; Raven, P.B. Classification of sports. J.Am. Coll. Cardiol. 1994, 24, $864-866$. [CrossRef]

33. Dlin, R.A.; Benmair, J.; Hanne, N. Pain Relief in Sports Injuries-Application of TENS to Acupuncture Points. Int. J. Sports Med. 1980, 1, 203-206. [CrossRef]

34. Sternfeld, M.; Finkelstein, Y.; Eliraz, A.; Hod, I. Runner's stitch syndrome successfully treated by acupuncture. Am. J. Acupunct. 1992, 20, 213-215.

35. Seplow, W. Management of Lateral Meniscus Injury. Sports Chiropr. Rehabil. 1996, 10, 86-88.

36. Hoven, J.; Snyder, R.; Andrew, T. Management of peroneal nerve entrapment in an elite skier: A case report. J. Sports Chiropr. Rehabil. 2000, 14, 70-74.

37. Rosted, P. Acupuncture for treatment of the yips?-A case report. Acupunct. Med. 2005, 23, 188-189. [CrossRef]

38. Young, K. Sports Injuries and TCM. J. Chin. Med. 2005, 78, 1-10.

39. Martinez-Silvestrini, J.A.; Micheo, W.F. Complex regional pain syndrome in pediatric sports: A case series of three young athletes. Boletín la Asoc Médica Puerto Rico 2006, 98, 31-37. 
40. Chen, Y.Z.; Xu, Y.; Yang, W.B.; Lu, Y.H.; Fang, H.B. Observation on therapeutic effect of catgut implantation at acupoints on exercise fatigue and study on the mechanism. Zhongguo Zhen Jiu 2008, 28, 656-658.

41. Yan, X.N.; Ren, C.X. Cause and treatment of sports injury in knee joint medial collateral ligament. Zhongguo Zuzhi Gongcheng Yanjiuyu Linchuang Kangfu 2008, 12, 5567-5570.

42. Osborne, N.J.; Gatt, I.T. Management of Shoulder Injuries Using Dry Needling in Elite Volleyball Players. Acupunct. Med. 2010, 28, 42-45. [CrossRef]

43. Donoyama, N.; Hotoge, S.; Ohkoshi, N. Acupuncture Might Have Contributed to Improving Amenorrhoea in a Top Athlete. Acupunct. Med. 2011, 29, 304-306. [CrossRef] [PubMed]

44. Yuill, E.A.; Pajaczkowski, J.A.; Howitt, S.D. Conservative care of sports hernias within soccer players: A case series. J. Bodyw. Mov. Ther. 2012, 16, 540-548. [CrossRef]

45. Morimoto, Y.; Saito, A.; Tokuhashi, Y. Low level laser therapy for sports injuries. Laser Ther. 2013, 22, 17-20. [CrossRef] [PubMed]

46. Pearcey, G.E.P.; Bradbury-Squires, D.J.; Power, K.E.; Behm, D.G.; Button, D.C. Exertional Rhabdomyolysis in an Acutely Detrained Athlete/Exercise Physiology Professor. Clin. J. Sport Med. 2013, 23, 496-498. [CrossRef]

47. Woitzik, E.; Kissel, J. Ganglion cyst of the foot treated with electroacupuncture: A case report. J. Can. Chiropr. Assoc. 2013, 57, 310-315.

48. Abe, G.C.; Yonamine, B.T.S.; Mello, C.A.D.S.; Ramos, P.E.; Conde, M.S.; Oliveira, A.S.B. Acupuncture for Treating Persistent Pain in Brazilian Para-Athletes. Med Acupunct. 2014, 26, 50-56. [CrossRef]

49. Gliedt, J.A.; Daniels, C.J. Chiropractic management of elbow tendinopathy following a sports related trauma. J. Can. Chiropr. Assoc. 2014, 58, 52-57.

50. Jayaseelan, D.J.; Moats, N.; Ricardo, C.R. Rehabilitation of Proximal Hamstring Tendinopathy Utilizing Eccentric Training, Lumbopelvic Stabilization, and Trigger Point Dry Needling: 2 Case Reports. J. Orthop. Sports Phys. Ther. 2014, 44, 198-205. [CrossRef] [PubMed]

51. Kleinhenz, J.; Streitberger, K.; Windeler, J.; Güssbacher, A.; Mavridis, G.; Martin, E. Randomised clinical trial comparing the effects of acupuncture and a newly designed placebo needle in rotator cuff tendinitis. Pain 1999, 83, 235-241. [CrossRef]

52. Kamali, F.; Sinaei, E.; Morovati, M. Comparison of Upper Trapezius and Infraspinatus Myofascial Trigger Point Therapy by Dry Needling in Overhead Athletes With Unilateral Shoulder Impingement Syndrome. J. Sport Rehabil. 2019, 28, 243-249. [CrossRef]

53. Callison, M. Clinical study: Acupuncture \& tibial stress syndrome (Shin splints). J. Chin. Med. 2002, 70, 24-28.

54. Karagounis, P.; Tsironi, M.; Prionas, G.; Tsiganos, G.; Baltopoulos, P. Treatment of plantar fasciitis in recreational athletes: Two different therapeutic protocols. Foot Ankle Spec. 2011, 4, 226-234. [CrossRef]

55. Timpka, T.; Jacobsson, J.; Bickenbach, J.; Finch, C.F.; Ekberg, J.; Nordenfelt, L. What is a sports injury? Sports Med. 2014, 44, 423-428. [CrossRef]

56. Joseph, C.W.; Finch, C.F. Sports Injuries. International Encyclopedia of Public Health, 2nd ed.; Quah, S.R., Cockerham, W.C., Eds.; Academic Press: Cambridge, MA, USA, 2017; pp. 79-86.

57. Renström, P.; Johnson, R.J. Overuse injuries in sports. A review. Sports Med. 1985, 2, 316-333. [CrossRef]

58. Crockett, B. Rehabilitation of the athlete. Mo. Med. 2011, 108, 173-175.

59. Babatunde, O.O.; Jordan, J.L.; Van der Windt, D.A.; Hill, J.C.; Foster, N.E.; Protheroe, J. Effective treatment options for musculoskeletal pain in primary care: A systematic overview of current evidence. PLoS ONE 2017, 12, e0178621. [CrossRef]

60. Mehallo, C.J.; Drezner, J.A.; Bytomski, J.R. Practical management: Nonsteroidal antiinflammatory drug (NSAID) use in athletic injuries. Clin. J. Sport Med. 2006, 16, 170-174. [CrossRef]

61. Jeon, M.S.; Bae, S.Y. The Study of Athlete Patients Visited Korean Medical Clinic in CheongJu-Gym at the 2016 CheongJu World Martial Arts. J. Sports Korean Med. 2016, 16, 51-56.

62. Oh, M.J.; Son, I.M.; Shin, J.M.; Cho, J.H. The Study of Patients Visited Korean Medical Clinic in Athletes' Village Hospital at The 28th Univesiade Gwanju Korea. J. Sports Korean Med. 2016, 16, 1-7.

63. Yang, C.; Lee, E.; Hwang, E.H.; Kwon, O.; Lee, J.H. Management of Sport Injuries with Korean Medicine: A Survey of Korean National Volleyball Team. Evid. Based Complement. Alternat. Med. 2016, 2016, 1-7. [CrossRef] 
64. Kim, D.S.; Lee, Y.H.; Bae, K.S.; Baek, G.H.; Lee, S.Y.; Shim, H.; On, M.G.; Rhie, S.J.Y. PyeongChang 2018 Winter Olympic Games and athletes' usage of 'polyclinic' medical services. BMJ Open Sport Exerc. Med. 2019, 5, e000548. [CrossRef]

65. Araujo, C.G.; Scharhag, J. Athlete: A working definition for medical and health sciences research. Scand. J. Med. Sci. Sports 2016, 26, 4-7. [CrossRef]

66. Chang, W.D.; Chang, N.J.; Lin, H.Y.; Wu, J.H. Effects of Acupuncture on Delayed-Onset Muscle Soreness: A Systematic Review and Meta-Analysis. Evid. Based Complement. Alternat. Med. 2020, 2020, 5864057. [CrossRef]

Publisher's Note: MDPI stays neutral with regard to jurisdictional claims in published maps and institutional affiliations.

(C) 2020 by the authors. Licensee MDPI, Basel, Switzerland. This article is an open access article distributed under the terms and conditions of the Creative Commons Attribution (CC BY) license (http://creativecommons.org/licenses/by/4.0/). 